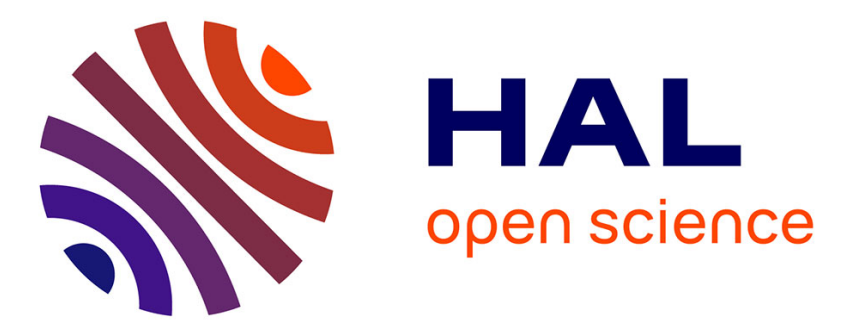

\title{
Confined viscoplastic flows with heterogeneous wall slip
}

Pandelitsa Panaseti, Anne-Laure Vayssade, Georgios Georgiou, Michel Cloitre

\section{To cite this version:}

Pandelitsa Panaseti, Anne-Laure Vayssade, Georgios Georgiou, Michel Cloitre. Confined viscoplastic flows with heterogeneous wall slip. Rheologica Acta, 2017, 56 (6), pp.539-553. 10.1007/s00397-0171016-1 . hal-02406307

\section{HAL Id: hal-02406307 https://hal.science/hal-02406307}

Submitted on 8 Jun 2021

HAL is a multi-disciplinary open access archive for the deposit and dissemination of scientific research documents, whether they are published or not. The documents may come from teaching and research institutions in France or abroad, or from public or private research centers.
L'archive ouverte pluridisciplinaire HAL, est destinée au dépôt et à la diffusion de documents scientifiques de niveau recherche, publiés ou non, émanant des établissements d'enseignement et de recherche français ou étrangers, des laboratoires publics ou privés. 


\title{
Confined viscoplastic flows with heterogeneous wall slip
}

\author{
Pandelitsa Panaseti \\ Department of Mathematics and Statistics, University of Cyprus \\ P.O. Box 20537, 1678 Nicosia, Cyprus \\ Anne-Laure Vayssade \\ MMN, Laboratoire Gulliver, CNRS, ESPCI Paris, PSL Research University, \\ 10 rue Vauquelin, 75005 Paris, France \\ Georgios C. Georgiou ${ }^{1}$ \\ Department of Mathematics and Statistics, University of Cyprus \\ P.O. Box 20537, 1678 Nicosia, Cyprus \\ Michel Cloitre \\ Soft Matter and Chemistry, CNRS, ESPCI Paris, PSL Research University \\ 10 Rue Vauquelin, 75005 Paris, France \\ 1: Corresponding author, E-mail: georgios@ucy.ac.cy, Tel. +357292612, Fax: \\ $+35722895352$
}

\begin{abstract}
The steady, pressure-driven flow of a Herschel-Bulkley fluid in a microchannel is considered assuming that different power-law slip equations apply at the two walls due to slip heterogeneities, allowing the velocity profile to be asymmetric. Three different flow regimes are observed as the pressure gradient is increased. Below a first critical pressure gradient $G_{1}$ , the fluid moves unyielded with a uniform velocity and thus the two slip velocities are equal. In an intermediate regime between $G_{1}$ and a second critical pressure gradient $G_{2}$, the fluid yields in a zone near the weak-slip wall and flows with uniform velocity near the stronger-slip wall. Beyond this regime, the fluid yields near both walls and the velocity is uniform only in the central unyielded core. It is demonstrated that the central unyielded region tends towards the midplane only if the power-law exponent is less than unity; otherwise, this region rends towards the weak-slip wall, and asymmetry is enhanced. The extension of the different flow regimes depends on the channel gap; in particular the intermediate asymmetric flow regime dominates when the gap becomes smaller than a characteristic length which incorporates the wall slip coefficients and the fluid properties. The theoretical results compare well with available experimental data on soft glassy suspensions. These results open new routes in manipulating the flow of viscoplastic materials in applications where the flow behavior depends not only on the bulk rheology of the material but also on the wall properties.
\end{abstract}


Keywords: Plane Poiseuille flow; Herschel-Bulkley model; Bingham plastic; Wall slip; Navier slip.

\section{Introduction}

Viscoplastic or yield-stress materials constitute a very interesting class which includes materials of industrial importance, such as polymeric solutions, suspensions and gels, but also biofluids like blood (Barnes 1999). These materials behave as fluids if the stress exceeds the yield stress, $\tau_{0}$, and as solids otherwise. Therefore, the constitutive equation of an ideal viscoplastic material consists of two branches. Let us denote the viscous stress tensor by $\tau$ and the rate of strain tensor by $\dot{\gamma}$, the latter being defined by

$$
\dot{\gamma} \equiv \nabla \mathbf{u}+(\nabla \mathbf{u})^{T}
$$

where $\mathbf{u}$ is the velocity vector and the superscript $T$ denotes the transpose. The magnitudes of $\dot{\gamma}$ and $\tau$, denoted respectively by $\dot{\gamma}$ and $\tau$, are defined by $\dot{\gamma} \equiv \sqrt{I I_{\dot{\gamma}} / 2}$ and $\tau \equiv \sqrt{I I_{\tau} / 2}$. As mentioned above, in those areas of the flow field where $\tau \leq \tau_{0}$ the fluid is unyielded, i.e. there is no flow and $\dot{\gamma}=\mathbf{0}$; otherwise the fluid is yielded and $\dot{\gamma} \neq \mathbf{0}$. The Herschel-Bulkley constitutive equation is widely used to describe the shear rheology of many viscoplastic materials (Herschel and Bulkley 1926):

$$
\begin{cases}\dot{\gamma}=\mathbf{0}, & \tau \leq \tau_{0} \\ \tau=\left(\frac{\tau_{0}}{\dot{\gamma}}+k \dot{\gamma}^{n-1}\right) \dot{\gamma}, & \tau>\tau_{0}\end{cases}
$$

where $k$ is the consistency index and $n$ is the power-law exponent. For viscoplastic materials made of soft and deformable particles such as microgel particles or emulsion droplets, the power-law exponent $n$ is close to 0.5 (Cloitre et al. 2003; Bécu et al. 2006; Ovarlez et al. 2008; Seth et al. 2011). The consistency index $k$ has been found to be proportional to the storage modulus of the suspensions (Seth et al. 2011). By setting $n=1$ and $k=\mu$, where $\mu$ is the plastic viscosity, the Bingham-plastic constitutive equation is recovered (Bingham 1922). By taking $\tau_{0}=0$, one gets the power-law model:

$$
\boldsymbol{\tau}=k \dot{\gamma}^{n-1} \dot{\gamma}
$$

Viscoplastic materials are prone to slip when they are sheared near smooth surfaces (Barnes 2005; Cloitre and Bonnecaze 2016). As a consequence of the solid-liquid duality of yield 
stress materials, slip essentially occurs at low shear rates below or near the yield point in contrast to slip of polymer melts which takes place at large shear rates (Denn 2001). This generic feature is shared by many particulate materials such as highly-filled suspensions (Yilmazer and Kalyon 1989; Kalyon 2005), microgel suspensions (Meeker et al. 2004a; Meeker et al. 2004b; Aktas et al. 2014; Ortega-Avila et al. 2016), concentrated emulsions (Princen 1985; Salmon et al. 2003; Seth et al. 2012), hard-sphere suspensions (Ballesta et al. 2008; 2012), and colloidal gels (Ballesta et al. 2013). At the microscopic scale, slip is due to the formation of a thin layer of liquid adjacent to the walls, which lubricates the contacts between the bulk suspension and the walls (Barnes 1995; Cloitre and Bonnecaze 2016). At the macroscopic scale, slip can be characterized using a power-law slip equation, relating the wall shear stress, $\tau_{w}$, to the slip velocity, $u_{w}$, defined as the relative velocity of the fluid with respect to that of the wall (Kalyon 2005):

$$
\tau_{w}=\beta u_{w}^{s}
$$

where $s$ is the slip exponent and $\beta$ is the slip coefficient. The latter coefficient incorporates the effects of several material properties affecting slip, such as the solvent viscosity and the particle properties. The no-slip and full-slip limiting cases are recovered in the limits $\beta \rightarrow \infty$ and $\beta=0$, respectively. The classical Navier slip condition (Navier 1823) is the special case of Eq. (4) for $s=1$ :

$$
\tau_{w}=\beta u_{w}
$$

in which case $\beta$ is related to the slip or extrapolation length $b$, i.e. $\beta \equiv \eta / b$, where $\eta$ is the fluid viscosity.

The slip exponent $s$ depends on the properties of the lubricated films at the material/wall interface. Two main lubrication mechanisms have been identified (Seth et al. 2008; 2012) . In simple hydrodynamic lubrication (HL), the wall is wetted by a thin film of solvent, which has a constant thickness independent of the flow velocity. For a Newtonian solvent, $s$ is equal to 1 ; for a non-Newtonian solvent with a power-law viscosity $\eta=k \dot{\gamma}^{m-1}, s$ is equal to $1 / m$ (Kalyon 2005). HL slip has been observed with highly-filled suspensions (Kalyon 2005), soft particle suspensions with repulsive particle-wall interactions (Seth et al. 2008; 2012; PérezGonzález et al. 2012), and hard-sphere glasses (Ballesta et al. 2008;2012). In elastohydrodynamic lubrication (EHL), the lubricating film results from a coupling between flow in the lubricating film and particle deformation. EHL is relevant in jammed suspensions 
of soft particles in the presence of slightly attractive particle-wall interactions (Meeker et al. 2004a; 2004b; Seth et al. 2018; 2012). Below the yield stress, $s$ is expected to be of the order of 2, which is observed in experiments (Meeker et al. 2004a; 2004b; Ortega-Avila et al. 2016; Ahonguio et al. 2016). Above the yield stress there exist no predictions and the situation is less clear. However experimental observations generally converge to the value $s=1$ (Aktas et al. 2014; Seth et al. 2012; Vayssade et al. 2014; Poumaere et al. 2014).

In real situations, complex fluids rarely flow in ideal geometries bounded by uniform surfaces but rather experience important slip heterogeneities induced by local variations of surface roughness and chemistry. In spite of its practical importance, this problem has retained little attention so far. Lauga and Stone analysed theoretically how surface heterogeneities, which were either transverse or parallel to the flow direction, affect the slip length of Newtonian fluids (Lauga and Stone 2003). In their study of the extrusion of viscoplastic suspensions in shallow channels, Lawal and Kalyon considered a Couette-Poiseuille model subject to different Navier-slip coefficients at the barrel and screw surfaces (Lawal and Kalyon 1994). The authors derived analytical solutions for the Couette-Poiseuille flow for the case where the imposed pressure gradient and the moving upper plate drive the flow in opposite directions. Different flow regimes were found depending on the velocity, both in magnitude and direction, of the upper wall. Recently Vayssade et al. imaged the motion of wellcharacterized soft glassy suspensions in microfluidic channels whose walls imposed different slip velocities (Vayssade et al. 2014). The rheology of the suspensions was well represented by a Herschel-Bulkley equation with $n=1 / 2$ and the boundary slip conditions were of the Navier form, i.e. $s=1$. It was found that, when the channel gap was large, the velocity profiles consisted of a central unyielded plug between two fluidized layers near the walls. When the gap was small, a remarkable behavior appeared: the fluidized layer adjacent to the wall with the highest slip velocity disappeared and the plug flow region extended down the wall.

These observations motivate the present work. We revisit the plane Poiseuille flow of a Herschel-Bulkley fluid with asymmetric wall slip, i.e. with different slip conditions at the two walls, in order to determine the critical conditions for the transition to different flow regimes when the degree of confinement varies. We successfully recover the experimental observations and provide quantitative criteria to get a particular flow regime and guidelines to predict confined flows of viscoplastic materials in the presence of strong surface heterogeneities. The paper is organized as follows. The governing equations and the general solution are presented in Section 2. Three different flow regimes are identified, which are 
defined by the two critical values of the imposed pressure gradient at which the fluid yields at each wall. In Section 3 we provide the analytical solutions for the flow of a power-law fluid subject to asymmetric Navier slip, which are very useful in interpreting the behavior of Herschel-Bulkley fluids at high pressure gradients (the power-law fluid can be viewed as the limit of a Herschel-Bulkley fluid as the pressure gradient goes to infinity). Section 4 is concerned with the variation of the critical pressure gradients with the gap size and the construction of flow diagrams. In Section 5, we make comparisons with the experimental data of Vayssade et al. (2014).

\section{General solutions for the asymmetric slip problem}

We consider the laminar, steady, unidirectional pressure-driven flow of a Herschel-Bulkley fluid in a horizontal channel of width $H$, as illustrated in Fig. 1. Heterogeneous wall slip is assumed to occur at the walls according to

$$
\tau_{w i}=\beta_{i} u_{w i}^{s}, \quad i=1,2
$$

where the lower and upper walls correspond to $i=1$ and 2, respectively. For the sake of simplicity, the slip exponents are considered to be the same at both walls. Since the flow is not symmetric, the origin is placed at the lower plate (Fig. 1). Without loss of generality, it is assumed that slip at the upper wall is stronger than at the lower wall, i.e. $\beta_{1} \geq \beta_{2}$ and thus $u_{w 2} \geq u_{w 1}$. With the above assumptions, the $x$-momentum equation for any generalized Newtonian fluid is simplified to:

$$
\frac{\partial \tau_{y x}}{\partial y}=-G
$$

which yields:

$$
\tau_{y x}=-G y+\tau_{w 1}
$$

where $G$ is the imposed pressure gradient. The lower-wall shear stress, $\tau_{w 1}$, is a crucial parameter, in terms of which all other quantities of interest can be expressed. The upper-wall shear stress is given by:

$$
\tau_{w 2}=\left|\tau_{y x}\right|_{y=H}=G H-\tau_{w 1}
$$

The two slip velocities $u_{w 1}$ and $u_{w 2}$ can then be calculated by means of Eq. (6).

For viscoplastic flow, we encounter the three regimes illustrated in Fig. 2 as the imposed pressure gradient is increased. In Regime I, the fluid simply slips and the velocity is constant (full-slip). This regime extends from zero up to the critical value $G_{1}$ of the pressure gradient 
at which the fluid adjacent to the lower wall (where slip is weaker) yields. Regime II extends from $G_{1}$ up to the critical value $G_{2}$ of the pressure gradient at which the fluid adjacent to the upper wall (where slip is stronger) also yields. Hence in Regime II only the lower layer of the fluid up to $y=y_{1}$ is yielded. In Regime III, i.e. for pressure gradients above $G_{2}$, the fluid yields near both walls and the velocity profile is asymmetric with a plug core between the lower and the upper yield points, $y_{1}$ and $y_{2}$. It is clear that Regime I is not relevant in the special case where there is no slip along the lower wall $\left(u_{w 1}=0\right)$ and Regime II is observed only if the flow is asymmetric $\left(\beta_{2}>\beta_{1}\right)$. In the following analysis we introduce the dimensionless slip numbers:

$$
B_{i} \equiv \frac{k^{s / n}}{\beta_{i} H^{s} \tau_{0}^{s / n-1}}, \quad i=1,2
$$

With this definition we have $B_{1} / B_{2}=\beta_{2} / \beta_{1}$ so that $B_{1} \leq B_{2}$ under our assumptions. Note that there is no slip at the wall when $B_{i}=0$.

\subsection{Regime I $\left(0 \leq G \leq G_{1}\right)$}

In Regime I, the pressure gradient is not sufficient to cause yielding of the material. However, since slip occurs along both walls and the material is unyielded, the two slip velocities are equal, $u_{w 1}=u_{w 2}$, and the material moves with uniform velocity:

$$
u_{x}(y)=\left(\frac{G H}{\beta_{1}+\beta_{2}}\right)^{1 / s}
$$

The lower wall shear stress, given by

$$
\tau_{w 1}=\frac{\beta_{1} G H}{\beta_{1}+\beta_{2}}
$$

is greater than or equal to $\tau_{w 2}$ since $\beta_{1} \geq \beta_{2}$. The critical pressure gradient $G_{1}$ which marks the transition between Regimes I and II is reached when the material adjacent to the lower wall yields $\left(\tau_{w 1}=\tau_{0}\right)$ :

$$
G_{1}=\left(1+\frac{B_{1}}{B_{2}}\right) \frac{\tau_{0}}{H}
$$

$G_{1}$ depends only on the yield stress and not on the exponent and consistency index. The maximum slip velocity is attained when $G=G_{1}$ :

$$
u_{x}=\left(\frac{\tau_{w 1}}{\beta_{1}}\right)^{1 / s}=\left(\frac{\tau_{0}}{\beta_{1}}\right)^{1 / s}
$$




\subsection{Regime II ( $\left.G_{1} \leq G \leq G_{2}\right)$}

This flow regime exists only if the flow is asymmetric, i.e. if $\beta_{1}>\beta_{2}$ (or $B_{1}<B_{2}$ ). In this case, the material yields only close to the lower wall, i.e. for $0 \leq y \leq y_{1}$, where $y_{1}$ is the yield point (Fig. 2), and remains unyielded for $y_{1}<y \leq H$, moving with uniform velocity equal to the upper slip velocity $u_{w 2}$. The yield point $y_{1}$ is found from Eq. (8) by demanding that $\tau_{y x}=\tau_{0}$. Hence,

$$
y_{1}=\frac{\tau_{w 1}-\tau_{0}}{G}
$$

In the yielded region $\left(0 \leq y \leq y_{1}\right)$,

$$
\tau_{y x}=\tau_{0}+k\left(\frac{d u_{x}}{d y}\right)^{n}=-G y+\tau_{w 1}
$$

Integrating the above equation and demanding that $u_{x}(0)=u_{w 1}$ and $u_{x}(y)=u_{x}\left(y_{1}\right)=u_{w 2}$, one finds that

$$
u_{x}(y)= \begin{cases}u_{w 1}+\frac{n G^{1 / n}}{(n+1) k^{1 / n}}\left[y_{1}^{1 / n+1}-\left(y_{1}-y\right)^{1 / n+1}\right], & 0 \leq y \leq y_{1} \\ u_{w 1}+\frac{n G^{1 / n}}{(n+1) k^{1 / n}} y_{1}^{1 / n+1}, & y_{1} \leq y \leq H\end{cases}
$$

Requiring that $u_{x}\left(y_{1}\right)=u_{w 2}$ leads to the following equation for the lower-wall shear stress:

$$
\frac{n}{n+1}\left(\tau_{w 1}-\tau_{0}\right)^{1 / n+1}+\left[\left(B_{1} \tau_{w_{1}}\right)^{1 / s}-\left[B_{2}\left(G H-\tau_{w_{1}}\right)\right]^{1 / s}\right] \tau_{0}^{1 / n-1 / s} G H=0
$$

The second critical pressure gradient $G_{2}$ signals the yielding of the fluid at the upper wall and therefore it can be found by demanding that $\tau_{w 2}=\tau_{0}$, which leads to:

$$
\frac{n}{n+1}\left(G H-2 \tau_{0}\right)^{1 / n+1}-\left[\left(B_{2} \tau_{0}\right)^{1 / s}-\left[B_{1}\left(G H-\tau_{0}\right)\right]^{1 / s}\right] \tau_{0}^{1 / n-1 / s} G H=0
$$

\subsection{Regime III $\left(G>G_{2}\right)$}

In this regime, there are two yielded regions adjacent to the two walls separated by an intermediate unyielded region $\left(y_{1} \leq y \leq y_{2}\right)$. The solution derived above for $0 \leq y \leq y_{1}$ in Regime II still applies. The second yield point is given by: 


$$
y_{2}=\frac{\tau_{w_{1}}+\tau_{0}}{G}
$$

and the three-branch velocity profile reads:

$$
u_{x}(y)= \begin{cases}u_{w 1}+\frac{n G^{1 / n}}{(n+1) k^{1 / n}}\left[y_{1}^{1 / n+1}-\left(y_{1}-y\right)^{1 / n+1}\right], & 0 \leq y \leq y_{1} \\ u_{w 1}+\frac{n G^{1 / n}}{(n+1) k^{1 / n}} y_{1}^{1 / n+1}, & y_{1} \leq y \leq y_{2} \\ u_{w 2}+\frac{n G^{1 / n}}{(n+1) k^{1 / n}}\left[\left(H-y_{2}\right)^{1 / n+1}-\left(y-y_{2}\right)^{1 / n+1}\right], & y_{2} \leq y \leq H\end{cases}
$$

By demanding that $u_{x}\left(y_{1}\right)=u_{x}\left(y_{2}\right)$, one finds the following equation for the lower-wall shear stress:

$$
\frac{n}{n+1}\left[\left(\tau_{w_{1}}-\tau_{0}\right)^{1 / n+1}-\left(G H-\tau_{w_{1}}-\tau_{0}\right)^{1 / n+1}\right]+\left[\left(B_{1} \tau_{w_{1}}\right)^{1 / s}-\left[B_{2}\left(G H-\tau_{w_{1}}\right)\right]^{1 / s}\right] \tau_{0}^{1 / n-1 / s} G H=0
$$

\section{Solutions for power-law fluids}

\subsection{Non-dimensional equations}

In this section we solve the asymmetric slip equations for the special case of power-law fluids described by Eq. (3). Given that the power-law flow can be viewed as the limiting case of the Herschel-Bulkley flow at infinite pressure gradient, the results derived below will be useful in understanding the flow of Herschel-Bulkley fluids at high values of the pressure gradient. It is interesting to note that asymmetric flow profiles have also been observed with polymer solutions which are well represented by a power-law constitutive equation (MüllerMohnssen et al. 2007). In the limit of a power-law fluid, the two yield points $y_{1}$ and $y_{2}$ collapse to the unique point $y_{M}$, where the velocity attains its maximum. The yield stress is zero and therefore there is no natural scale for stresses. The governing equations can be made dimensionless by scaling lengths by $H$, the pressure gradient by an arbitrary value, say $G_{\text {s }}$, so that $G^{*} \equiv G / G_{s}$, stresses by $G_{s} H$, and the velocity by $G_{s}^{1 / n} H^{1+1 / n} / k^{1 / n}$. The slip numbers are redefined as

$$
B_{i} \equiv \frac{k^{s / n}}{\beta_{i} H^{s / n+s-1} G_{s}^{s / n-1}}, \quad i=1,2
$$

The non dimensional velocity profiles are then given by: 
$u_{x}^{*}\left(y^{*}\right)=\left\{\begin{array}{cc}u_{w 1}^{*}+\frac{n G^{* 1 / n}}{n+1}\left[y_{M}^{* 1 / n+1}-\left(y_{M}^{*}-y^{*}\right)^{1 / n+1}\right] & 0 \leq y^{*} \leq y_{M}^{*} \\ u_{w 2}^{*}+\frac{n G^{* 1 / n}}{n+1}\left[\left(1-y_{M}^{* 1 / n}\right)-\left(y^{*}-y_{M}^{*}\right)^{1 / n+1}\right] & y_{M}^{*} \leq y^{*} \leq 1\end{array}\right.$

The position of the maximum velocity $y_{M}^{*}$ is a root of

$$
\frac{n}{n+1} G^{* 1 / n-1 / s}\left[y_{M}^{* 1 / n+1}-\left(1-y_{M}^{*}\right)^{1 / n+1}\right]+B_{1}^{1 / s} y_{M}^{* 1 / s}-B_{2}^{1 / s}\left(1-y_{M}^{*}\right)^{1 / s}=0
$$

If $B_{1}=B_{2}$, the flow is symmetric and $y_{M}^{*}=1 / 2$ for any value of the pressure gradient $G^{*}$. When $n=s, y_{M}^{*}$ is independent of $G^{*}$ and can be found by solving

$$
\frac{n}{n+1}\left[y_{M}^{*_{1 / n+1}}-\left(1-y_{M}^{*}\right)^{1 / n+1}\right]+B_{1}^{1 / n} y_{M}^{* 1 / n}-B_{2}^{1 / n}\left(1-y_{M}^{*}\right)^{1 / n}=0
$$

For the particular case of Newtonian flow with Navier slip, i.e. for $n=s=1$, one finds

$$
y_{M}^{*}=\frac{1+2 B_{2}}{2\left(1+B_{1}+B_{2}\right)}, \quad n=s=1
$$

When $n \neq s$, the position of the maximum changes as the pressure gradient is increased. If $n<s$, it is easily shown that $y_{M}^{*}$ decreases asymptotically to $1 / 2$ starting from a finite value $y_{0}^{*}:$

$$
y_{0}^{*} \equiv \lim _{G^{*} \rightarrow 0} y_{M}^{*}=\frac{B_{2}}{B_{1}+B_{2}}, \quad y_{\infty}^{*} \equiv \lim _{G^{*} \rightarrow \infty} y_{M}^{*}=\frac{1}{2}, \quad n<s
$$

If $n>s$, the two limits are reversed. Hence, for Navier slip $(s=1)$,

$$
y_{0}^{*}=\left\{\begin{array}{ll}
\frac{B_{2}}{B_{1}+B_{2}}, & n<1 \\
\frac{1+2 B_{2}}{2\left(1+B_{1}+B_{2}\right)}, & n=1, \\
\frac{1}{2}, & n>1
\end{array} \quad y_{\infty}^{*}= \begin{cases}\frac{1}{2}, & n<1 \\
\frac{1+2 B_{2}}{2\left(1+B_{1}+B_{2}\right)}, & n=1 \\
\frac{B_{2}}{B_{1}+B_{2}}, & n>1\end{cases}\right.
$$

\subsection{Discussion}

The evolution of the velocity profiles as the pressure gradient is increased is shown in Fig. 3. In the first two columns of Fig. 3, we consider a shear thickening fluid $(n=4 / 3)$, a Newtonian fluid $(n=1)$, and a shear thinning fluid $(n=1 / 2)$, following Navier-slip laws $(s=1)$ with $B_{2}=2$ and $B_{1}=0$ and 2 , respectively. With shear-thinning fluids the velocity profiles tend to become more symmetric as the pressure gradient increases, whereas with shear-thickening fluids asymmetry is amplified. By comparing the first two columns of Fig. 3, we see that this 
effect becomes more pronounced when the fluid sticks at the lower wall $\left(B_{1}=0\right)$. The position of the maximum of the velocity profiles decreases from $y_{0}^{*}$ to $y_{\infty}^{*}=1 / 2$ when the fluid is shear-thinning and increases from $y_{0}^{*}=1 / 2$ to $y_{\infty}^{*}$ when the fluid is shear-thickening. Similar trends are shown in the third column of Fig. 3 where a power-law slip equation with $s=1 / 2$ is used. The second row of Fig. 3 shows that $y_{M}^{*}$ is independent of the pressure gradient when $n=s$. Finally, in the third row of Fig. 3 we observe that when $n<s$ the velocity profiles tend to become symmetric with $y_{M}^{*}$ decreasing asymptotically to $1 / 2$ as the pressure gradient is increased.

\section{Solutions for Herschel-Bulkley fluids}

\subsection{Non-dimensional equations}

The asymptotic results discussed above are useful in understanding the flow of HerschelBulkley fluids at high values of the pressure gradient. The velocity profiles are computed from the general equations established in Section 2. It is convenient to scale lengths by $H$, stresses by $\tau_{0}$, pressure gradient by $G_{\mathrm{s}}=\tau_{0} / H$ and velocity by $u_{\mathrm{s}}=H\left(\tau_{0} / k\right)^{1 / n}$. The critical dimensionless pressure gradients $G_{1}^{*}$ and $G_{2}^{*}$ are then given by:

$$
G_{1}^{*} \equiv 1+\frac{B_{1}}{B_{2}} \text { and } \frac{n}{n+1}\left(G_{2}^{*}-2\right)^{1 / n+1}-\left[B_{2}^{1 / s}-B_{1}^{1 / s}\left(G_{2}^{*}-1\right)^{1 / s}\right] G_{2}^{*}=0
$$

The velocity profiles, the yield points and the wall stresses in each regime are provided below.

\section{Regime I}

$$
\begin{aligned}
& u_{x}^{*}\left(y^{*}\right)=\left(\frac{B_{1} B_{2} G^{*}}{B_{1}+B_{2}}\right)^{1 / s} \\
& \tau_{w 1}^{*}=\frac{B_{2} G^{*}}{B_{1}+B_{2}}
\end{aligned}
$$

\section{Regime II}




$$
\begin{aligned}
& u_{x}^{*}\left(y^{*}\right)= \begin{cases}u_{w 1}^{*}+\frac{n G^{* 1 / n}}{n+1}\left[y_{1}^{* 1 / n+1}-\left(y_{1}^{*}-y^{*}\right)^{1 / n+1}\right], & 0 \leq y^{*} \leq y_{1}^{*} \\
u_{w 1}^{*}+\frac{n G^{* 1 / n}}{n+1} y_{1}^{* 1 / n+1}, & y_{1}^{*} \leq y^{*} \leq 1\end{cases} \\
& \frac{n}{n+1}\left(\tau_{w 1}^{*}-1\right)^{1 / n+1}+\left[\left(B_{1} \tau_{w_{1}}^{*}\right)^{1 / s}-B_{2}^{1 / s}\left(G^{*}-\tau_{w_{1}}^{*}\right)^{1 / s}\right] G^{*}=0 \\
& y_{1}^{*}=\frac{\tau_{w 1}^{*}-1}{G^{*}}
\end{aligned}
$$

\section{Regime III}

$$
\begin{aligned}
& u_{x}^{*}\left(y^{*}\right)= \begin{cases}u_{w 1}^{*}+\frac{n G^{* 1 / n}}{n+1}\left[y_{1}^{*_{1 / n+1}}-\left(y_{1}^{*}-y^{*}\right)^{1 / n+1}\right], & 0 \leq y^{*} \leq y_{1}^{*} \\
u_{w 1}^{*}+\frac{n G^{* 1 / n}}{n+1} y_{1}^{* 1 / n+1}, & y_{1}^{*} \leq y^{*} \leq y_{2}^{*} \\
u_{w 2}^{*}+\frac{n G^{* 1 / n}}{n+1}\left[\left(1-y_{2}^{*}\right)^{1 / n+1}-\left(y^{*}-y_{2}^{*}\right)^{1 / n+1}\right], & y_{2}^{*} \leq y^{*} \leq 1\end{cases} \\
& \frac{n}{n+1}\left[\left(\tau_{w 1}^{*}-1\right)^{1 / n+1}-\left(G^{*}-\tau_{w 1}^{*}-1\right)^{1 / n+1}\right]+\left[\left(B_{1} \tau_{w_{1}}^{*}\right)^{1 / s}-B_{2}^{1 / s}\left(G^{*}-\tau_{w_{1}}^{*}\right)^{1 / s}\right] G^{*}=0 \\
& y_{1}^{*}=\frac{\tau_{w 1}^{*}-1}{G^{*}} \quad \text { and } y_{2}^{*}=\frac{\tau_{w 1}^{*}+1}{G^{*}}
\end{aligned}
$$

In the general case, the second critical pressure gradient $G_{2}^{*}$ and the lower wall shear stress $\tau_{w 1}^{*}$, in terms of which the solution is expressed, can only be calculated numerically. In the case of Navier slip $(s=1)$, these quantities can be calculated analytically for certain values of $n$. The Bingham plastic flow $(n=1)$ belongs to this category and the corresponding solutions are given in Appendix B.

\subsection{Velocity profiles}

Figure 4 shows velocity profiles for different values of the pressure gradient covering the three regimes, obtained for four different values of the exponent $(n=4 / 3,1,1 / 2$ and $1 / 3)$ assuming Navier slip $(s=1)$ with $B_{1}=1$ and $B_{2}=2$. Recall that the velocity profiles in Regime I are independent of the exponent $n$ and that $u_{x}^{*}\left(G_{1}^{*}\right)=B_{1}$ in all cases. Just as for the power-law fluids, shear thickening tends to enhance the flow asymmetry, in the sense that both yield points move away from the midplane towards the upper wall, while shear thinning tends to favor symmetry and extends Regime II. As expected, both yield points tend to 
converge to the asymptotic point $y_{\infty}^{*}$ given by Eq. (29), which corresponds to the power-law solution. More specifically, the yield points for $n=4 / 3$ converge to $y_{\infty}^{*}=2 / 3$ (Fig. $4 \mathrm{a}$ ), those for $n=1$ to $y_{\infty}^{*}=5 / 8$ (Fig. $4 \mathrm{~b}$ ), while those for $n=1 / 2$ and $1 / 3$ converge to $y_{\infty}^{*}=1 / 2$ (Figs. $4 \mathrm{c}$ and d). It is interesting to note that the variations of $y_{1}^{*}$ and $y_{2}^{*}$ with the pressure gradient are not always monotonic. For example, $y_{1}^{*}$ is a monotonically increasing function of the pressure gradient only for large values of $n$ (Figs. 4a-c). When $n=1 / 3$ (Fig. 4d) $y_{1}^{*}$ initially increases attaining a maximum and then decreases over a wide range of the pressure gradient before starting increasing again to asymptotically reach the value $y_{\infty}^{*}=1 / 2$. Similarly, when $n=4 / 3$ (Fig. $4 \mathrm{a}), y_{2}^{*}$ decreases and reaches a minimum before increasing asymptotically to the limiting value $y_{\infty}^{*}=2 / 3$ far from the midplane.

\subsection{Wall stress and slip velocity}

The effect of the pressure gradient on the wall shear stresses and the slip velocities is illustrated in Fig. 5. Here, based on the behavior of soft glassy suspensions (Vayssade et al. 2014), we consider a Herschel-Bulkley material with exponent $n=1 / 2$ and assume that Navier slip $(s=1)$ occurs along both walls. The lower plate slip number takes two extreme values, i.e. $B_{1}=0.001$ and 1 , corresponding to weak and strong slip respectively; the ratio $B_{1} / B_{2}=0.5$ is fixed. From Eq. (29), we have $G_{1}^{*}=1.5$ showing that Regime I is the same in all cases; $G_{2}^{*}$ increases and Regime II expands as $B_{1}$ is increased. In Fig. 5, we observe that the variations of the slip velocities with the pressure gradient are correlated with the variations of the wall shear stresses. For weak slip (Fig. 5a), the difference between $\tau_{w 1}^{*}$ and $\tau_{w 2}^{*}$ increases in Regime I, decreases in Regime II, and finally vanishes in Regime III where eventually $\tau_{w 1}^{*}=$ $\tau_{w 2}^{*}$. The corresponding velocities $u_{w 1}^{*}$ and $u_{w 2}^{*}$ increase as the pressure gradient is increased. These are equal in Regime I but in the other two regimes $u_{w 2}^{*}$ grows faster than $u_{w 1}^{*}$ and thus the difference $u_{w 2}^{*}-u_{w 1}^{*}$ increases. Strong slip (Fig. 5b) results in higher slip velocities as expected. Both $\tau_{w 1}^{*}$ and $\tau_{w 2}^{*}$ increase but the rate of increase is slowly changing over the entire range of pressure gradient explored. For a given value of the slip ratio, the difference of the two slip velocities in Regimes II and III is much smaller and its rate of increase is much lower for strong slip than for weak slip. 


\section{Confined flows with asymmetric slip}

\subsection{General solution}

In this section we show that for a given viscoplastic material and fixed wall properties, the critical pressure gradients marking the onset of Regimes II and III, depend on the gap size $H$. In order to analyze this dependence, we need to introduce new length, pressure-gradient and velocity scales:

$$
\ell_{s}=\frac{k^{1 / n}}{\tau_{0}^{1 / n-1 / s} \beta_{2}^{1 / s}}, \quad G_{s}=\frac{\tau_{0}^{1+1 / n-1 / s} \beta_{2}^{1 / s}}{k^{1 / n}}, \quad \text { and } \quad u_{s}=\left(\frac{\tau_{0}}{\beta_{2}}\right)^{1 / s}
$$

The resulting dimensionless variables are denoted by a tilde $(\sim)$. It is also important to note that the slip equation parameters $\beta_{2}$ and $s$ (along the upper wall) are hidden in the nondimensional scales (38). In particular, the non dimensional gap $\tilde{H} \equiv H / \ell_{s}$ incorporates the effects of the slip coefficient at the upper wall and the fluid properties. From Eq. (13) we get the following dimensionless expression for the first critical pressure gradient:

$$
\tilde{G}_{1}=\frac{1+\kappa}{\tilde{H}}
$$

where

$$
\kappa \equiv \frac{\beta_{2}}{\beta_{1}}=\frac{B_{1}}{B_{2}}
$$

is the slip parameter ratio which varies from 0 (no slip along the lower wall) to 1 (same slip along the wall). Hence the curve representing $\tilde{G}_{1}$ versus $\tilde{H}$ lies between $1 / \tilde{H}$ and $2 / \tilde{H}$. The second critical pressure gradient $\tilde{G}_{2}$ is the root of the dimensionless version of Eq. (19):

$$
(\tilde{G} \tilde{H}-2)^{1 / n+1}-\left(1+\frac{1}{n}\right)\left[1-\kappa^{1 / s}(\tilde{G} \tilde{H}-1)^{1 / s}\right] \tilde{G}=0
$$

The above equation is amenable to analytical solution only in some special cases. Two of them of practical interest in experiments are discussed below.

\subsection{Solution for no slip along the lower wall $(\kappa=0)$}

In this case, $\tilde{G}=1 / \tilde{H}$ and Eq. (42) is simplified to

$$
\left(\tilde{G}_{2} \tilde{H}-2\right)^{1 / n+1}=\left(1+\frac{1}{n}\right) \tilde{G}_{2}
$$

For a Bingham fluid $(n=1)$ one finds 


$$
\tilde{G}_{2}=\frac{2}{\tilde{H}}+\frac{1}{\tilde{H}^{2}}(1+\sqrt{1+4 \tilde{H}})
$$

while for a Herschel-Bulkley fluid with $n=1 / 2$,

$$
\tilde{G}_{2}=\frac{2}{\tilde{H}}\left\{1+\frac{1}{\sqrt{\tilde{H}}} \cos \left[\frac{1}{3} \cos ^{-1}(3 \sqrt{\tilde{H}})\right]\right\}
$$

It is useful to note that if $G_{2}$ and $H$ are known from experiments and if the upper wall experiences Navier slip $(s=1)$ then the slip coefficient can be calculated from the dimensional version of Eq. (43):

$$
\beta_{2}=\frac{(1+1 / n) k^{1 / n} G}{\tau_{0}^{1 / n}\left(G H / \tau_{0}-2\right)^{1 / n+1}}
$$

The first and second critical pressure gradients for $n=1$ and $1 / 2$ are plotted as functions of $\tilde{H}$ in Fig. 6. These graphs can be viewed as flow diagrams giving the type of flow experienced by the fluid when the degree of confinement is varied. Regime I is situated below the curve $\tilde{G}_{1}=f(\tilde{H})$, which is independent of exponent $n$. Regime II is the area between the curves of $\tilde{G}_{1}$ and $\tilde{G}_{2}$. Figure 6 highlights the importance of the characteristic length $\ell_{\mathrm{s}}$ defined in (39). When the gap $H$ becomes lower than the characteristic length $\ell_{\mathrm{s}}(\widetilde{H}<1)$, Regime II dominates and Regime III is hardly attained except at very large pressure gradients. When $\tilde{H}$ increases, the fluid is less confined and the extension of Regimes I and II is reduced. As expected, when the fluid is more shear-thinning, i.e. $n$ is lower, the critical pressure gradient for yielding at the upper wall, $\tilde{G}_{2}$, is reduced and the extension of Regime II is reduced accordingly. At large values of $\tilde{H}, \tilde{G}_{2}$ becomes independent of $n$.

\subsection{Solution for Navier slip along both walls $(s=1)$}

In this case, Eq. (42) is simplified to

$$
(\tilde{G} \tilde{H}-2)^{1 / n+1}-\left(1+\frac{1}{n}\right)[1-\kappa(\tilde{G} \tilde{H}-1)] \tilde{G}=0
$$

For Bingham fluids $(n=1)$ one gets:

$$
\tilde{G}_{2}=\frac{1}{\tilde{H}+2 \kappa}\left\{2+\frac{1}{\tilde{H}}\left[1+\kappa+\sqrt{(1+\kappa)^{2}+4(1-\kappa) \tilde{H}}\right]\right\}
$$

For Herschel-Bulkley fluids with $n=1 / 2$

$$
\tilde{G}_{2}=\frac{2}{\tilde{H}}+\frac{1}{\tilde{H}^{2}}\left[\frac{(1-3 \kappa) \tilde{H}+\kappa^{2}}{C^{1 / 3}}+C^{1 / 3}-\kappa\right]
$$


where:

$$
C=\frac{1}{2}\left[6(1-\kappa) \tilde{H}^{2}+\left\{3 \kappa(3 \kappa-1)+\sqrt{36(1-\kappa)^{2} \tilde{H}^{2}+4\left(9 \kappa^{2}-1\right) \tilde{H}-3(1+\kappa)^{2} \kappa^{2}}\right\} \tilde{H}-2 \kappa^{3}\right]
$$

Figure 7 shows flow diagrams for Herschel-Bulkley fluids with $n=1 / 2$ and different values of the slip parameter ratio $\kappa$. Again the results exemplify the importance of the characteristic length $\ell$ s. Regime II has a significant extension when the gap $H$ is comparable to or lower than $\ell$ s. It is shifted upwards when $\kappa$ is reduced and reaches its maximum extension when $\kappa=0$. When $\kappa=1$, the critical pressure gradients $\tilde{G}_{1}$ and $\tilde{G}_{2}$ are equal to $2 / \tilde{H}$ indicating that Regime II disappears and the flow shifts directly from pure slip to upper and lower yielded flows

\section{Discussion}

In this section we relate our results with those of Vayssade et al. (2014) for the flow of Herschel-Bulkley glassy suspensions in microchannels.

\subsection{Quantifying the asymmetry of the velocity profiles}

\section{Definition of the asymmetry parameter}

Vayssade et al. (2014) solved the flow of Herschel-Bulkley fluid in microchannels by considering a frame of reference at the midplane of the channel, which translates at a mean velocity $\left(u_{w 1}+u_{w 2}\right) / 2$ so that the two walls move with opposite velocities $\pm U_{s}$, where:

$$
U_{s}=\frac{u_{w 2}-u_{w 1}}{2}
$$

They also defined the dimensionless number:

$$
S=\frac{1}{2}\left(\frac{y_{1}}{H}+\frac{y_{2}}{H}-1\right)
$$

as a measure of the asymmetry in the positions of the yield points. Their analysis focused on the two slip velocities only and was independent of the slip laws at the two walls. However, it was restricted to the particular case $n=1 / 2$. In this section, we revisit the notion of the asymmetry parameter to compare our results to the experimental data, taking advantage of the general solutions derived in Section 2. Generalizing the scaling forms proposed by Vayssade et al. (2014), we scale velocities by $n G^{1 / n} H^{1+1 / n} /(n+1) k^{1 / n}$, distances by $H$, pressure gradients by $\tau_{0} / H$ and stresses by $G H$. In order to avoid confusion with our previous adimensionalization systems, the dimensionless variables in this section are denoted 
by bars. According to Eq. (21), the difference between the two slip velocities in Regime III can be written in dimensionless form as:

$$
\bar{u}_{w 2}-\bar{u}_{w 1}=\bar{y}_{1}^{1 / n+1}-\left(1-\bar{y}_{2}\right)^{1 / n+1}
$$

or

$$
\bar{U}_{s}=\frac{1}{2}\left[\bar{y}_{1}^{1 / n+1}-\left(1-\bar{y}_{2}\right)^{1 / n+1}\right]
$$

For $n \leq 1$, we conveniently set $n=1 / m$, where $m$ is an integer, to get

$$
\bar{U}_{s}=\frac{1}{2}\left(\bar{y}_{1}+\bar{y}_{2}-1\right) \sum_{k=1}^{m+1} \bar{y}_{1}^{m+1-k}\left(1-\bar{y}_{2}\right)^{k-1}
$$

Let us now assume that despite the fact that the slip velocities are not the same, the two yield points are almost symmetric about the midplane, i.e. $\bar{y}_{1} \approx 1-\bar{y}_{2}$. As already discussed, this assumption is not reasonable when $n \geq s$ in which case the two yield points tend to merge at $\bar{y}_{\infty}>1 / 2$, according to Eq. (29). It is valid when $n<s$ and the two slip velocities are close to each other. Setting $1-\bar{y}_{2}=\bar{y}_{1}$ into Eq. (54) yields:

$$
\bar{U}_{s}=\frac{1}{2^{1 / n+1}}\left(1+\frac{1}{n}\right) \bar{y}_{1}^{1 / n}\left(\bar{y}_{1}+\bar{y}_{2}-1\right)
$$

For $n<s$ and sufficiently high values of the pressure gradient, $\bar{y}_{1}$ can be approximated by $\bar{y}_{\infty} \approx 1 / 2$ and by combining Eqs. (52) and (55) the asymmetry parameter takes the form:

$$
S=\frac{2^{1 / n}}{1+1 / n} \bar{U}_{s}
$$

\section{Asymptotic values of the asymmetry parameter}

The asymptotic value $S_{\infty} \equiv\left(2 \bar{y}_{\infty}-1\right) / 2$ of $S$ as the pressure gradient goes to infinity is of interest. For example, when $s=1$ one gets from Eq. (29):

$$
S_{\infty}=\bar{y}_{\infty}-\frac{1}{2}= \begin{cases}0, & n<1 \\ \frac{B_{2}-B_{1}}{2\left(1+B_{1}+B_{2}\right)}, & n=1 \\ \frac{B_{2}-B_{1}}{2\left(B_{1}+B_{2}\right)}, & n>1\end{cases}
$$


For Bingham fluids $(n=1)$, the asymptotic value of $\bar{U}_{s}$ can be calculated from the slip velocities in the Newtonian case, which are known:

$$
\bar{U}_{s \infty}=\frac{B_{2}-B_{1}}{2\left(1+B_{1}+B_{2}\right)}=S_{\infty}
$$

In the general case, when $s \neq 1, S_{\infty}$ is calculated by means of

$$
S_{\infty}=\frac{1}{2}\left(2 \bar{y}_{M}-1\right)
$$

where $\bar{y}_{M}$ is the position of the maximum velocity in the flow of a power-law fluid, which can be found by solving Eq. (26). $\bar{U}_{s \infty}$ is then found by means of Eq. (56), which has been derived by assuming that $\bar{y}_{\infty} \approx 1 / 2$.

\subsection{Relevance of the asymmetry parameter}

The expression (57) for $S_{\infty}$ is valid for high values of the pressure gradient and $n<s$. Let us test it against the exact solutions found in the previous sections. We consider the case $n=1 / 2$ with Navier slip $(s=1)$, which is representative of the experiments of Vayssade et al. (2014). Figure 8a shows results of $S$ versus $\bar{U}_{s}$ obtained for different values of the slip number $B_{2}$ and $B_{1}=1$ over a wide range of pressure gradients above $G_{2}^{*}$. It is important to note that for low values of the pressure gradient, $S$ is actually double-valued when plotted against $\bar{U}_{s}$. As the pressure gradient is increased further both $S$ and $\bar{U}_{s}$ are reduced and all the curves approach asymptotically the line $S=2 \bar{U}_{s}$. The asymmetry parameter $S$ increases as $B_{2}$ is increased (Fig. 8a) or as $B_{1}$ is reduced (data not shown), because the asymmetry of the velocity profile is enhanced.

The influence of the power-law exponent is illustrated in Fig. 8b, where results obtained for Navier slip, i.e. $s=1$, with $B_{1}=1$ and $B_{2}=2$ and $n=1,1 / 2$ and 1/4 are shown. A first observation is that in the Bingham-plastic case for which $n=s, S$ is a decreasing function of $\bar{U}_{s}$ and there is no branch approaching asymptotically the line $S=\bar{U}_{s}$ predicted by Eq. (56). Actually, as the pressure gradient is increased, the calculated values of $S$ and $\bar{U}_{s}$ converge to the point ( $\bar{U}_{s \infty}, S_{\infty}$ ) instead of approaching asymptotically the line $S=\bar{U}_{s}$. This is due to the fact that the asymptotic forms derived for $S$ are only valid for $n<s$. For low values of the pressure 
gradient the variation of $S$ is essentially the same for all values of the power-law exponent. When $n<s$ the curves of $S$ bend to approach asymptotically the lines predicted by Eq. (56).

\subsection{Comparison with experiments}

For the rest of this section we fix the values of all material parameters, again based on the experiments of Vayssade et al. (2014), $n=1 / 2, s=1$, and $\kappa \equiv B_{1} / B_{2}=0.53$, and vary the gap size, $H$, from 7 up to $100 \mu \mathrm{m}$. The first dimensionless slip number $B_{1}$ is in the range from $0.15(H=100 \mu \mathrm{m})$ to $3(H=7 \mu \mathrm{m})$. The critical pressure gradients $\bar{G}_{1}$ and $\bar{G}_{2}$ are equal to $G_{1}^{*}$ and $G_{2}^{*}$ given by Eq. (60). Hence, the first critical pressure gradient is $G_{1}^{*}=1.53$, independently of the gap size. The second critical pressure gradient $G_{2}^{*}$ ranges from 2.6534 $(H=100 \mu \mathrm{m})$ to $2.8619(H=7 \mu \mathrm{m})$. Figure 9 shows the velocity profiles corresponding to $G^{*}=3,4,5,6$, and 8 for $H=7,20,50$, and $100 \mu \mathrm{m}$. To facilitate the comparison with the results of Vayssade et al. (2014), we plot the reduced velocity $u_{x}-u_{w 1}$, where $u_{w 1}$ is the smallest slip velocity, versus $y^{*}=\bar{y}=y / H$. The two yield points in the velocity profiles are marked with red circles. Given that $n<s$, the yield points tend asymptotically to the center of the channel $\left(\bar{y}_{\infty}=1 / 2\right)$ as the pressure gradient is increased. Given the experimental uncertainties discussed below, the agreement between the computed velocity profiles and the experimental ones is quite satisfactory. Quantitatively the computed profile lay below the experimental profiles but we noted that a moderate uncertainty on the consistency parameter ( $\cong 10 \%$ ) can explain the discrepancy. Although all values of the pressure gradient should fall into Regime III, the velocity profiles expected for low pressure gradient exhibit the asymmetrical semi-plateau shape observed in experiments. Again, this can be associated with experimental uncertainties on the slip parameter, since it is delicate to control the preparation of the surfaces with a high accuracy. Moreover, the asymmetry significantly decreases when the pressure gradient and/or the gap size are increased. This unambiguously confirms that the observed velocity profiles result from confinement effects.

Let us now turn our attention towards the asymmetry parameter. For $n=1 / 2$, one easily derives the asymptotic limit valid for large pressure gradients: $S=4 \bar{U}_{s} / 3$, which is the expression (apart from a minor typo) derived by Vayssade et al. (2014). In Fig. 10, the theoretical variations of the asymmetry parameter $S$ with $\bar{U}_{s}$ are shown for the same gap sizes as in Fig. 9, together with the experimental data (which also include points obtained for $H=75$ and 80 $\mu \mathrm{m})$. All the curves collapse into the asymptotic limit expected for large pressure gradients, 
in excellent agreement with the corresponding experimental data. When the gap size increases, the asymmetry parameter increases as $B_{1}$ is reduced, and the asymmetry curves are shifted toward low values of $U_{\mathrm{s}}$ when the pressure gradient is small. The full symbols in Fig. 10 represent values of the asymmetry parameter either in Regime II or in the lower part of Regime III. This choice is justified by the fact that experimentally it is difficult to attribute unambiguously a velocity profile to a particular flow regime. Indeed Regime II is quite narrow so that some velocity profiles that look like having a semi plateau shape may well belong to Regime III theoretically. In any case, the values of $S$ in all these points are in the range from 0.1 to 0.5 . This can be explained by noting that the experimental asymmetry parameter in Regime II has been calculated by setting $\bar{y}_{2}=1$, i.e. assuming that $S_{I I}=\bar{y}_{1} / 2$. Given that $\bar{y}_{1}$ also tends to unity as the pressure gradient is reduced from $\bar{G}_{2}$ to $\bar{G}_{1}$ (at which the velocity is plug) the theoretical limiting value of $S_{I I}$ is 0.5 while the corresponding value of $\bar{U}_{s}$ vanishes, independently of the gap size. It should be pointed out, however, that the measured slip velocities (full symbols) in Fig. 10 are lower than their counterparts in the upper part of Regime III (open symbols), as it is easily deduced from Fig. 9, which implies that the relative error in $\bar{U}_{s}$ may be higher.

\section{Conclusions}

We have analyzed the plane Poiseuille flow of a Herschel-Bulkley fluid with asymmetric wall slip. Three different flow regimes have been identified by means of two critical pressure gradients $G_{1}$ and $G_{2}$ : (a) in Regime I ( $0 \leq G \leq G_{1}$ ), the two slip velocities are the same and the velocity is uniform; (b) in Regime II $\left(G_{1}<G \leq G_{2}\right)$, the fluid yields in a zone near the weak-slip wall and flows with uniform velocity near the stronger-slip wall; and (c) in Regime III ( $G>G_{2}$ ), the fluid yields near both walls and the velocity is uniform only in the central unyielded core. The asymptotic limit flow of the Herschel-Bulkley flow as the pressure gradient tends to infinity is simply the flow of a power-law fluid which was also analysed and shed light to the flow of interest. The theoretical results compare well with the experimental data of Vayssade et al. (2014) on soft glassy suspensions. One important finding concerns the asymmetry parameter $S$, defined in Eq. (52), which is multi-valued for low and moderate pressures (in Regime III) and thus should be used with caution in interpreting the experimental data. 
To close this discussion we would like to stress out that the phenomena analyzed in this paper constitute a new and interesting situation of non local rheology where the flow behavior is controlled by the surface and not only by the bulk rheology of the material. By changing the topography and the particle-wall interactions it is thus possible to manipulate the flow and get different velocity profiles. This is particularly important in real situations, for instance during oil migration in porous media, where surface roughness and chemistry locally vary so that slip heterogeneities naturally exist. Many other relevant applications concern confined flows in microfluidic devices where surface effects dominate: dispensing nozzles of colloidal inks in $3 \mathrm{D}$ printing systems, inkjet printing, and extrusion of complex fluids.

\section{Appendix A - General solution in Regime I}

In the general case with different slip exponents at the two walls

$$
\tau_{w i}=\beta_{i} u_{w i}^{s_{i}}, \quad i=1,2
$$

and the two slip velocities satisfy

$$
\beta_{1} u_{w 1}^{s_{1}}+\beta_{2} u_{w 2}^{s_{2}}=G H
$$

In Regime I, $u_{w 1}=u_{w 2}=u_{w}$ and thus

$$
\beta_{1} u_{w}^{s_{1}}+\beta_{2} u_{w}^{s_{2}}=G H
$$

After solving the above equation for $u_{w}$ we can calculate the two wall shear stresses by means of Eq. (A1). The first critical pressure gradient can be then found by setting the hydrophilic wall shear stress equal to the yield stress, $\tau_{w 1}=\tau_{0}$.

Independent experiments of Vayssade et al. (2014) on soft glassy suspensions showed that $s_{1}=1$ and $s_{2}=1 / 2$. From Eq. (A3) we get

$$
u_{w}=\frac{\beta_{2}^{2}}{4 \beta_{1}^{2}}\left(\sqrt{1+\frac{4 \beta_{1} G H}{\beta_{2}^{2}}}-1\right)^{2}
$$

The first critical pressure gradient is given by

$$
G_{1}=\left(1+\frac{\sqrt{\beta_{1} \tau_{0}}}{\beta_{2}}\right) \frac{\beta_{2} \sqrt{\tau_{0} / \beta_{1}}}{H}
$$

\section{Appendix B - Bingham-plastic flow with Navier slip}


It is clear from Eq. (13) that the first critical pressure $G_{1}$ required for the material to yield at the lower wall is independent of the consistency index and the power-law exponent. In the case of Bingham plastic flow with Navier slip $(n=s=1)$, Eq. (19) for the second critical pressure gradient (the pressure gradient at which the material adjacent to the upper wall yields) is simplified as follows:

$$
\frac{1}{2}\left(G H-2 \tau_{0}\right)^{2}-\left[\left(B_{1}+B_{2}\right) \tau_{0}-B_{1} G H\right] G H=0
$$

and thus $G_{2}$ is given by

$$
G_{2}=\frac{2 \tau_{0} / H}{1+\frac{B_{1}+B_{2}}{2}\left[1 \pm \sqrt{1+4\left(B_{2}-B_{1}\right) /\left(B_{1}+B_{2}\right)^{2}}\right]}
$$

The lower root is chosen if it is greater than $G_{1}$ and the higher one otherwise.

The lower-wall shear stress in the three regimes is given by

$$
\frac{\tau_{w 1}}{G H}= \begin{cases}\frac{B_{2}}{B_{1}+B_{2}}, & 0 \leq G \leq G_{1} \\ \frac{\tau_{0}}{G H}+\sqrt{\left(B_{1}+B_{2}\right)^{2}+2 B_{2}-2\left(B_{1}+B_{2}\right) \frac{\tau_{0}}{G H}}-B_{1}-B_{2}, & G_{1} \leq G \leq G_{2} \\ \frac{1+2 B_{2}-2 \tau_{0} / G H}{2\left(1+B_{1}+B_{2}-2 \tau_{0} / G H\right)}, & G_{2} \leq G\end{cases}
$$

The two slip velocities can be calculated by means of

$$
u_{w 1}=\frac{\tau_{w 1}}{\beta_{1}} \quad \text { and } \quad u_{w 2}=\frac{G H-\tau_{w 1}}{\beta_{2}}
$$

and the positions of the yield points by:

$$
\frac{y_{1}}{H}=\frac{\tau_{w 1}-\tau_{0}}{G H}=\left\{\begin{array}{lc}
\sqrt{\left(B_{1}+B_{2}\right)^{2}+2 B_{2}-2\left(B_{1}+B_{2}\right) \frac{\tau_{0}}{G H}}-B_{1}-B_{2}, & G_{1} \leq G \leq G_{2} \\
\frac{1+2 B_{2}-2 \tau_{0} / G H}{2\left(1+B_{1}+B_{2}-2 \tau_{0} / G H\right)}-\frac{\tau_{0}}{G H}, & G_{2} \leq G
\end{array}\right.
$$

and

$$
\frac{y_{2}}{H}=\frac{\tau_{w 1}+\tau_{0}}{G H}=\frac{1+2 B_{2}-2 \tau_{0} / G H}{2\left(1+B_{1}+B_{2}-2 \tau_{0} / G H\right)}+\frac{\tau_{0}}{G H}, \quad G_{2} \leq G
$$

Finally, the velocity in Regimes I-III is given respectively by 


$$
\begin{gathered}
u_{x}^{I}(y)=\frac{G H}{\beta_{1}+\beta_{2}}, \\
u_{x}^{I I}(y)= \begin{cases}u_{w 1}+\frac{G}{2 \mu}\left[y_{1}^{2}-\left(y_{1}-y\right)^{2}\right], & 0 \leq y \leq y_{1} \\
u_{w 1}+\frac{G}{2 \mu} y_{1}^{2}, & y_{1} \leq y \leq H\end{cases}
\end{gathered}
$$

and

$$
u_{x}^{I I I}(y)= \begin{cases}u_{w 1}+\frac{G}{2 \mu}\left[y_{1}^{2}-\left(y_{1}-y\right)^{2}\right], & 0 \leq y \leq y_{1} \\ u_{w 1}+\frac{G}{2 \mu} y_{1}^{2}, & y_{1} \leq y \leq y_{2} \\ u_{w 2}+\frac{G}{2 \mu}\left[\left(H-y_{2}\right)^{2}-\left(y-y_{2}\right)^{2}\right], & y_{2} \leq y \leq H\end{cases}
$$

The solution for the symmetric problem is obtained by setting $\beta_{1}=\beta_{2}$. The two critical pressure gradients are then equal, $G_{1}=G_{2}=2 \tau_{0} / H$, so that the intermediate Regime II disappears. Moreover, $\tau_{w 1}=\tau_{w 2}=G H / 2$ and the positions of the yield points in Regime III are given by:

$$
y_{1}=\frac{H}{2}-\frac{\tau_{0}}{G}, \quad y_{2}=\frac{H}{2}+\frac{\tau_{0}}{G}
$$

Hence, $u_{x}^{I}=G H /(2 \beta)$ while $u_{x}^{I I I}$ is given by Eq. (B9). 


\section{REFERENCES}

Ahonguio F, Jossic L, Magnin A (2016) Influence of slip on the flow of a yield stress fluid around a flat plate. AIChE J 62:1356-1363

Aktas S, Kalyon DM, Marín-Santibáñez BM, Pérez-González J (2014) Shear viscosity and wall slip behavior of a viscoplastic hydrogel. J Rheol 58:513-535

Ballesta P, Petekidis G, Isa L, Poon WCK, Besseling R (2008) Slip and flow of hard-sphere colloidal glasses. Phys Rev Lett 101:258201

Ballesta P, Petekidis G, Isa L, Poon WCK, Besseling R (2012) Wall slip and flow of concentrated hard-sphere colloidal suspensions. J Rheol 56:1005-1037

Ballesta P, Koumakis N, Besseling R, Poon WCK, Petekidis G (2013) Slip of gels in colloidpolymer mixtures under shear. Soft Matter 9:3237-3245

Barnes HA (1995) A review of the slip (wall depletion) of polymer solutions, emulsions and particle suspensions in viscometers: Its cause, character, and cure. J Non-Newtonian Fluid Mech 56: 221-251

Barnes HA (1999) The yield stress - a review or ' $\pi \alpha v \tau \alpha \rho \varepsilon$ ' - everything flows?. J NonNewtonian Fluid Mech 81: 133-178

Bécu L, Manneville S, Colin A (2006) Yielding and flow in adhesive and nonadhesive concentrated emulsions. Phys Rev Lett 96:138302

Bingham EC (1922) Fluidity and Plasticity. McGraw Hill, New-York

Cloitre M, Borrega R, Monti F, Leibler L (2003) Glassy dynamics and flow properties of soft colloidal pastes. Phys Rev Lett 90:068303

Cloitre M, Bonnecaze RT (2016) A review on wall slip in high solid dispersions. Rheol Acta submitted

Denn MM (2001) Extrusion instabilities and wall slip. Annu Rev Fluid Mech 33:265-287

Herschel W, Bulkley R (1926) Measurement of consistency as applied to rubber-benzene solutions. Proc Am Soc Test Mater 26:621-633

Kalyon DM (2005) Apparent slip and viscoplasticity of concentrated suspensions. J Rheol 49:621-640

Lauga E, Stone H (2003) Effective slip in pressure-driven Stokes flow. J Fluid Mech 489:5577

Lawal A, Kalyon DM (1994) Single screw extrusion of viscoplastic fluids subject to different slip coefficients at screw and barrel surfaces. Polymer Eng Sci 34:1471-1479

Meeker SP, Bonnecaze RT, Cloitre M (2004) Slip and flow of soft particle pastes. Phys Rev Lett 92:198302 
Meeker SP, Bonnecaze RT, Cloitre M (2004) Slip and flow in pastes of soft particles: direct observation and rheology. J Rheol 48:1295-1320

Müller-Mohnssen H, Löbl P, Schauerte W (2007) Direct determination of apparent slip for a ducted flow of polyacrylamide solutions. J Rheol 31:323-336

Navier CLMH (1823) Sur les lois du mouvement des fluides. Mem Acad R Sci Inst Fr 6:389440

Ortega-Avila JB, Pérez-González J, Marín-Santibáñez BM, Rodríguez-González F, Aktas S, Malik M, Kalyon DM (2016) Axial annular flow of a viscoplastic microgel with wall slip. J Rheol 60:503-515

Ovarlez G, Rodts S, Ragouilliaux A, Coussot P, Goyon J, Colin A (2008) Wide-gap Couette flows of dense emulsions: Local concentration measurements, and comparison between macroscopic and local constitutive law measurements through magnetic resonance imaging. Phys Rev E 78:036307

Pérez-González J, López-Durán JJ, Marín-Santibáñez BM, Rodríguez-González F (2012) Rheo-PIV of a yield-stress fluid in a capillary with slip at the wall. Rheol Acta 51:937-946 Poumaere A, Moyers-González M, Castelain C, Burghelea T (2014) Unsteady laminar flows of a Carbopol gel in the presence of wall slip. J Non-Newton Fluid Mech 205:28-40

Princen HM (1985) Rheology of foams and highly concentrated emulsions. II. Experimental study of the yield stress and wall effects for concentrated oil-in-water emulsions. J Colloid Interface Sci 105:150-171

Salmon JB, Bécu L, Manneville S, Colin A (2003) Towards local rheology of emulsions under Couette flow using Dynamic Light Scattering. Eur Phys J E 10:209-223

Seth J, Cloitre M, Bonnecaze RT (2008) Influence of short-range forces on wall-slip in microgel pastes. J Rheol 52:1241-1268

Seth JR, Mohan L, Locatelli-Champagne C, Cloitre M, Bonnecaze RT (2011) A micromechanical model to predict the flow of soft particle glasses. Nat Mater 10:838-843

Seth J, Locatelli-Champagne C, Monti F, Bonnecaze RT, Cloitre M (2012) How do soft particle glasses yield and flow near solid surfaces. Soft Matter 8:140-148

Vayssade A-L, Lee C, Terriac E, Monti F, Cloitre M, Tabeling P (2014) Dynamical role of slip heterogeneities in confined flows. Phys Rev E 89:052309

Yilmazer U, Kalyon DM (1989) Slip effects in capillary and parallel disk torsional flows of highly filled suspensions. J Rheol 33:1197-1212 


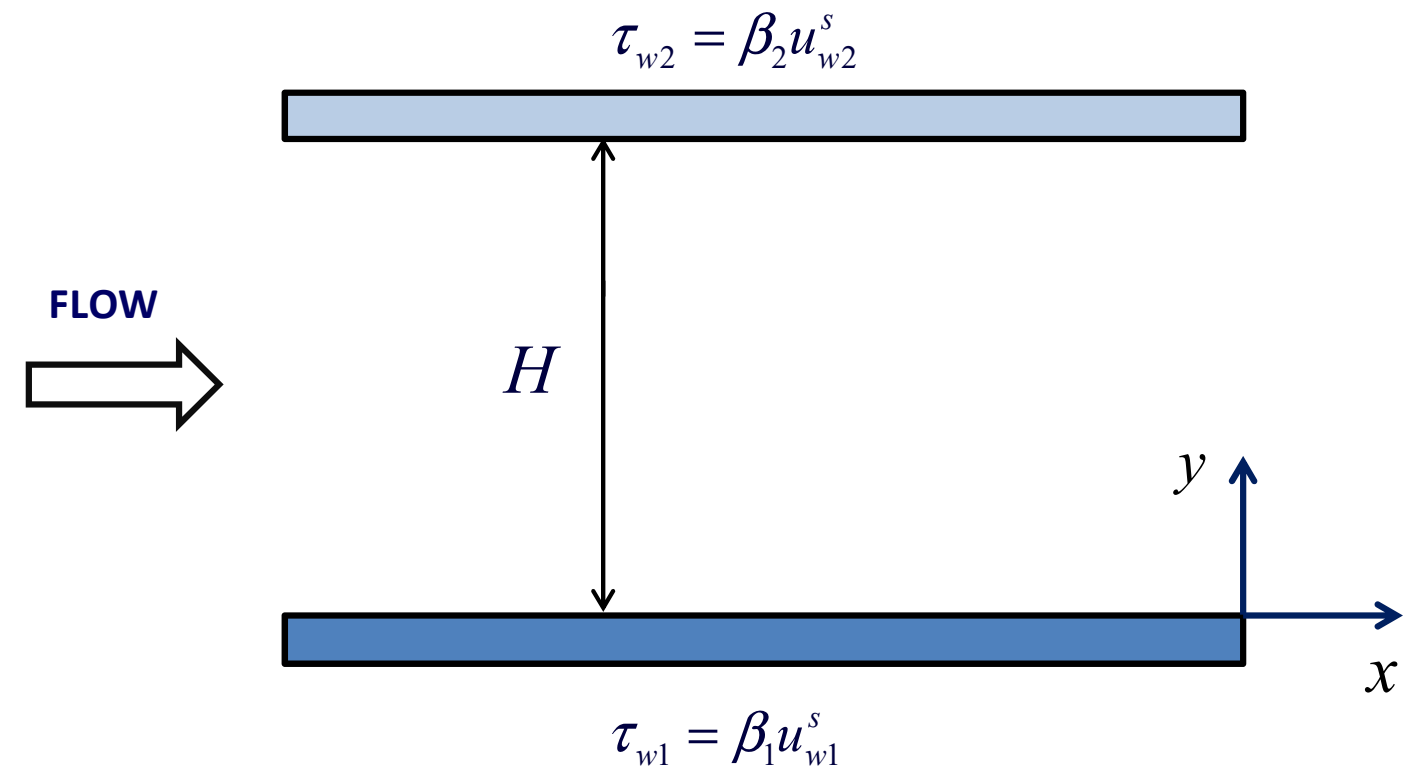

Figure 1. Geometry and boundary conditions of the flow development of a Herschel-Bulkley fluid in a channel with different slip laws at the walls.

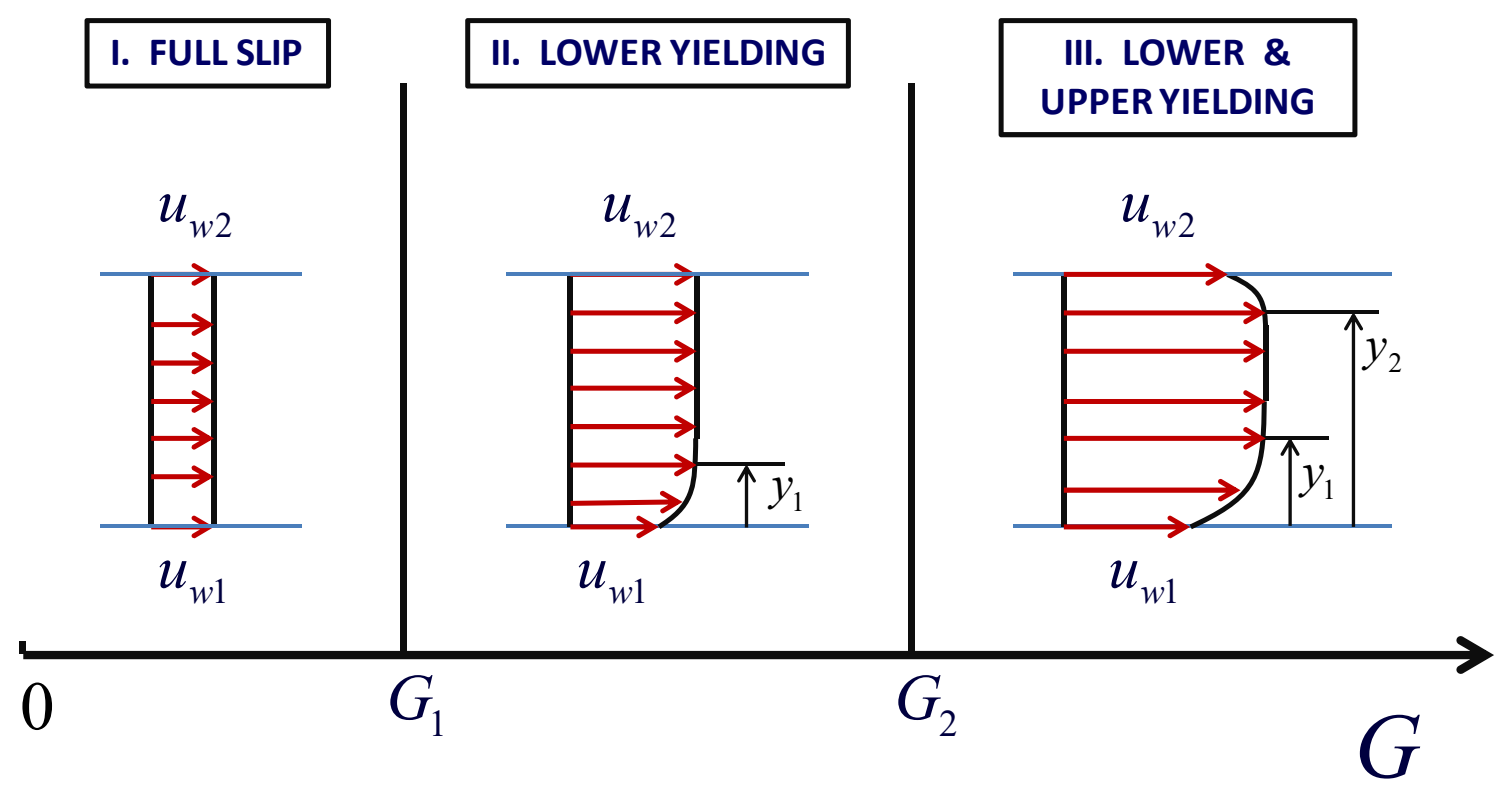

Figure 2. The three flow regimes encountered during viscoplastic Poiseuille flow with asymmetric slip. $y_{1}$ (and $y_{2}$ ) are the yield points which separate the yielded region(s) from the plug region and $u_{w 1}$ and $u_{w 2}$ are the slip velocities at the walls. It is assumed that slip is weaker at the lower plate ( $\left.u_{w 1}<u_{w 2}\right)$. 

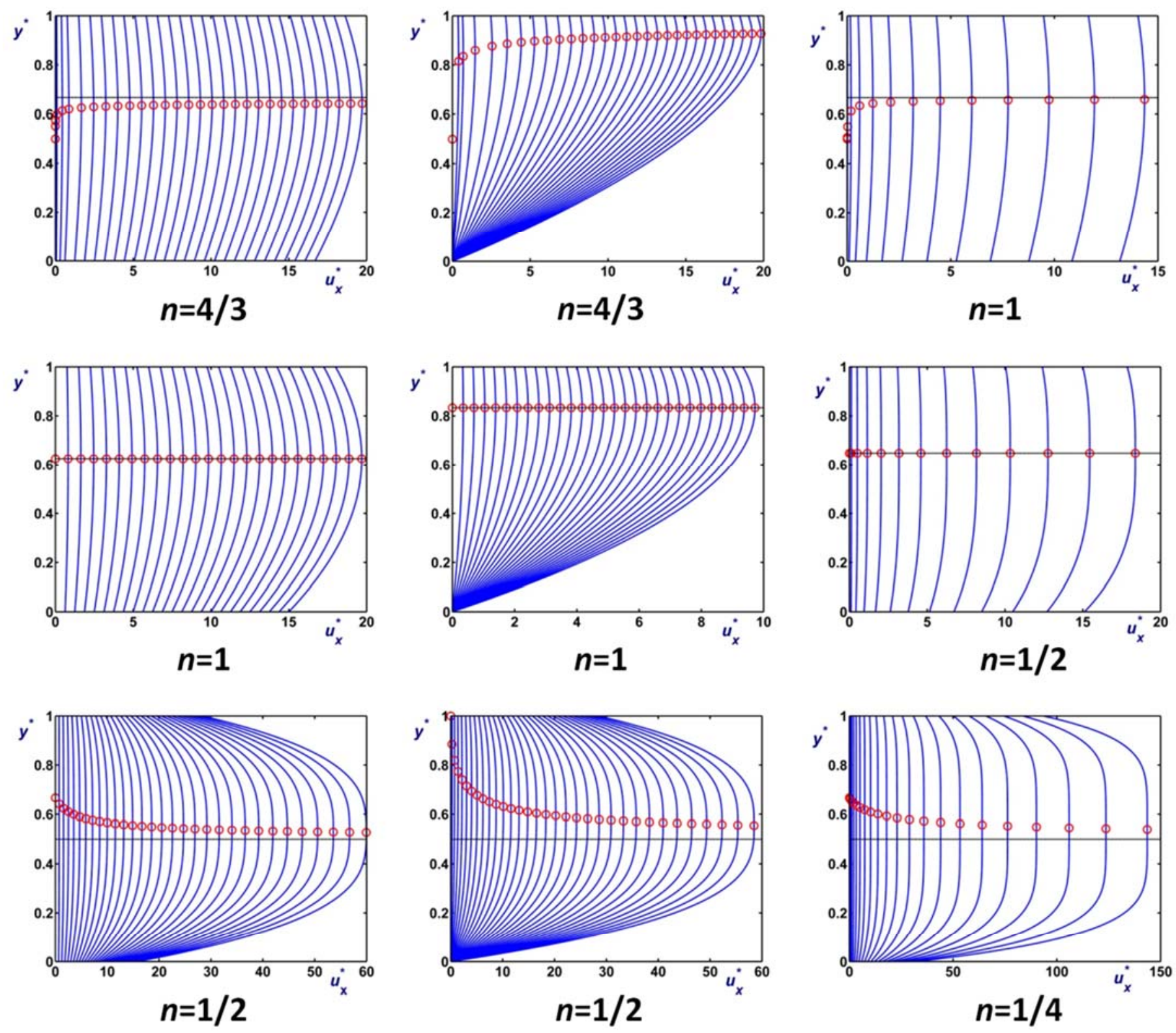

$$
s=1 ; B_{1}=1 \text { and } B_{2}=2
$$$$
s=1 ; B_{1}=0 \text { and } B_{2}=2
$$$$
s=1 / 2 ; B_{1}=1 \text { and } B_{2}=2
$$

Figure 3. Velocity profiles of various power-law fluids for different values of the pressure gradient, different slip laws $\left(s, B_{1}, B_{2}\right)$, and different power-law exponents $(n)$. The circles show the maximum velocity and the horizontal lines show the asymptotic values $y_{\infty}^{*}$. 


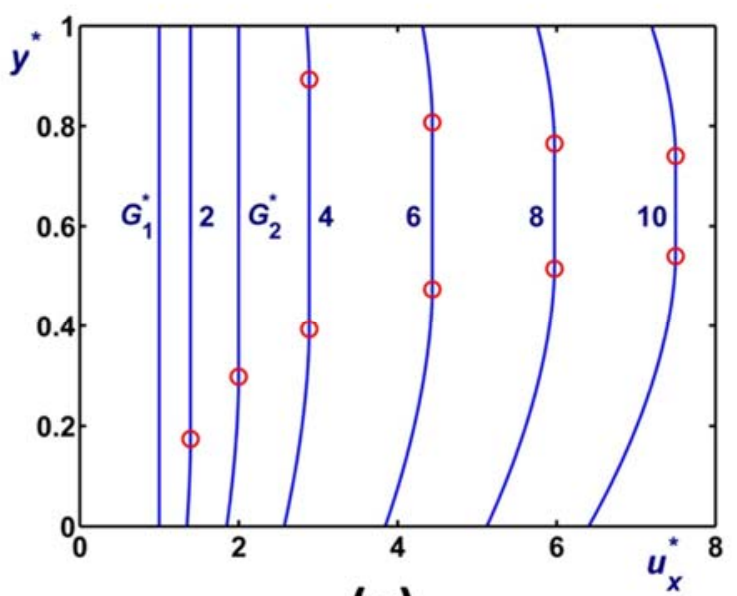

(a)

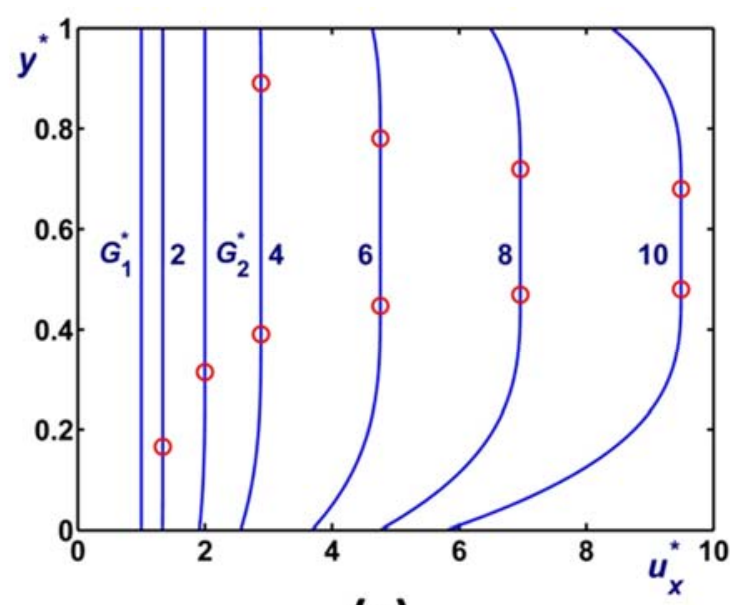

(c)

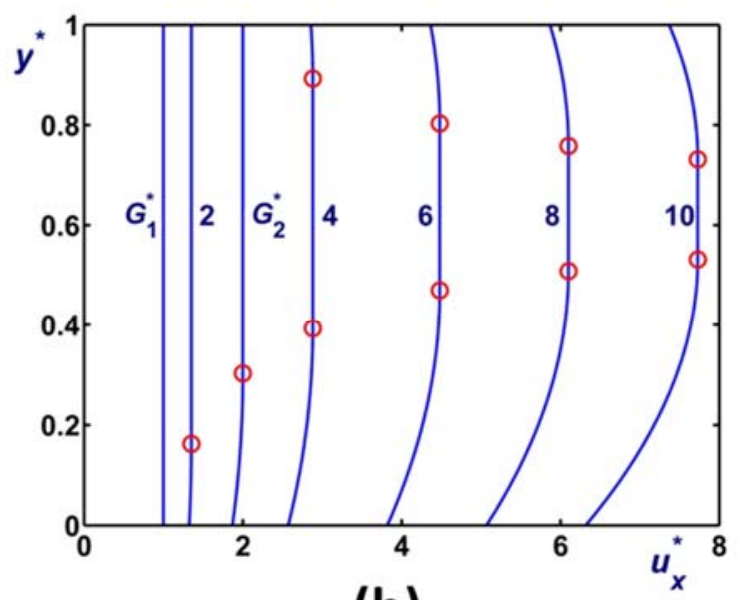

(b)

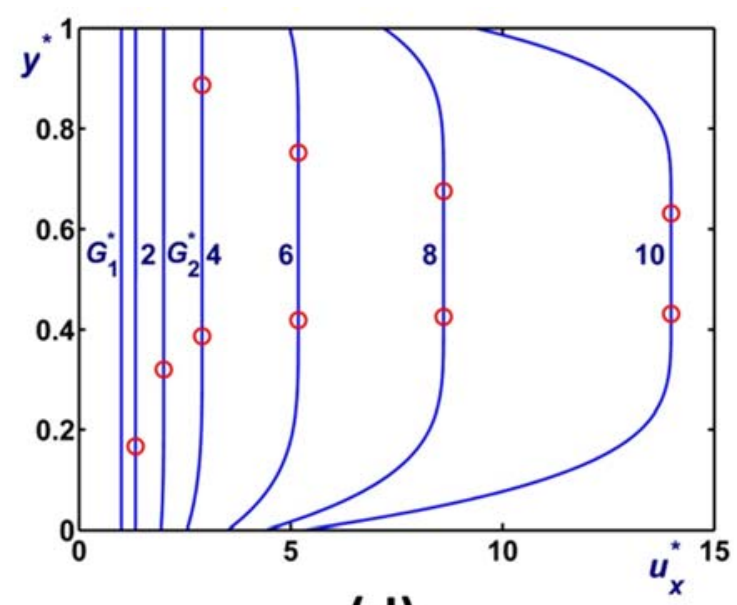

(d)

Figure 4. Velocity profiles of Herschel-Bulkley fluids for different values of the pressure gradient in the case of Navier slip (s=1) with $B_{1}=1$ and $B_{2}=2$ : (a) $n=4 / 3$ with $G_{2}^{*}=2.8943$ and $y_{\infty}^{*}=2 / 3$; (b) $n=1$ with $G_{2}^{*}=2.8685$ and $y_{\infty}^{*}=5 / 8$; (c) $n=1 / 2$ with $G_{2}^{*}=2.9129$ and $y_{\infty}^{*}=1 / 2$; (d) $n=1 / 3$ with $G_{2}^{*}=2.9349$ and $y_{\infty}^{*}=1 / 2$. Note that $G_{1}^{*}=1.5$ in all cases. Lengths $(y)$, velocities $\left(u_{\mathrm{x}}\right)$ and pressure gradients $(G)$ are scaled by $H, u_{0}=H /\left(\tau_{0} / k\right)^{1 / n}$ and $G_{s}=\tau_{0} / H$, respectively. The circles show the yield points. 

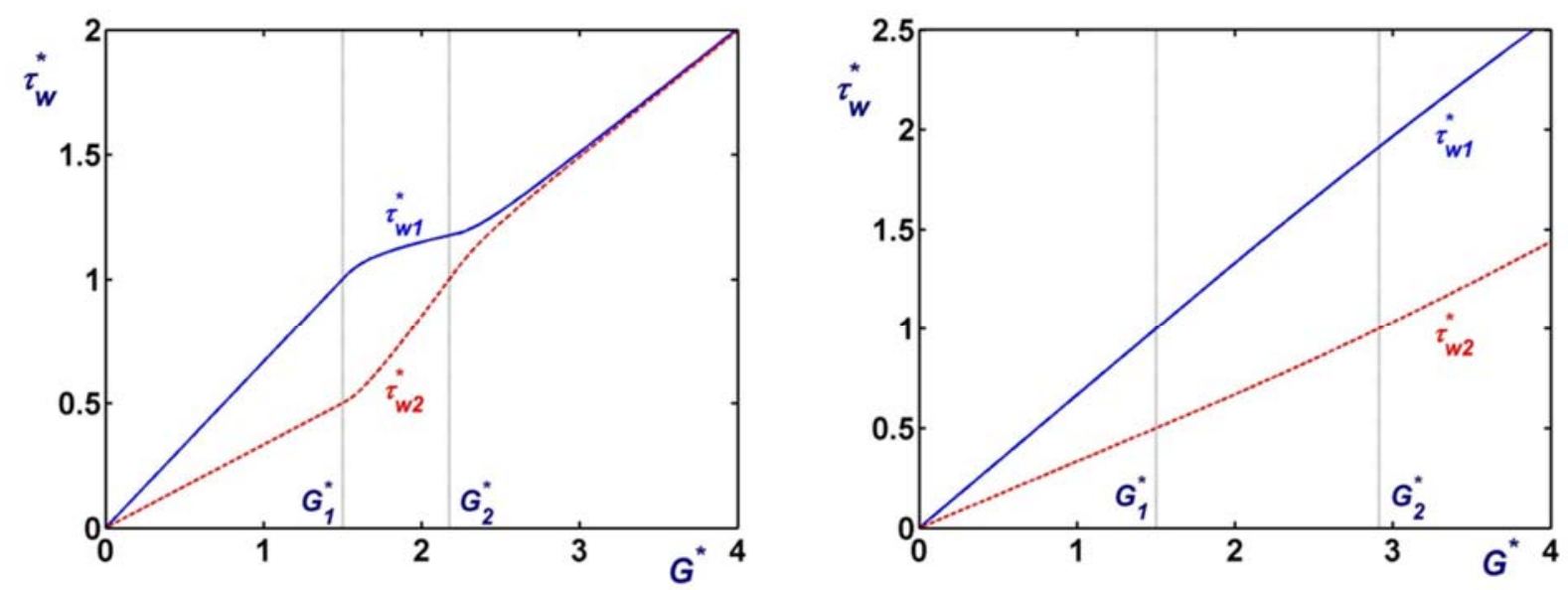

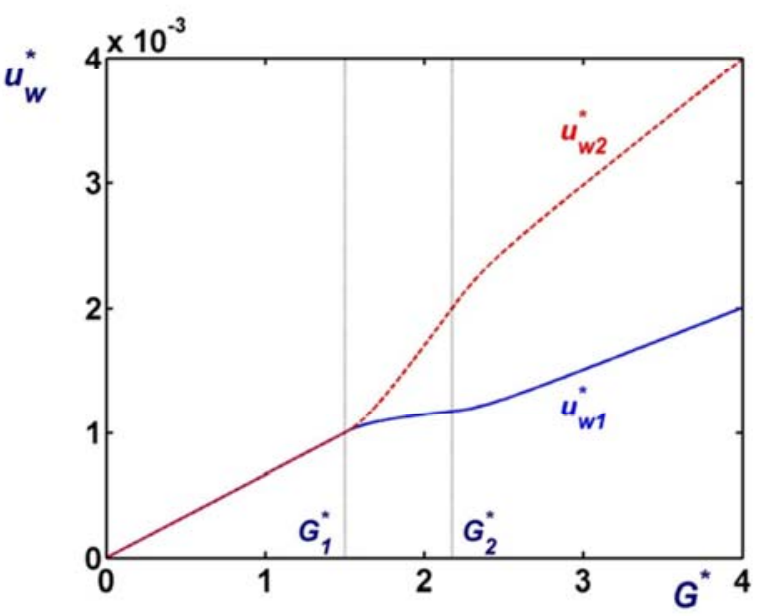

(a)

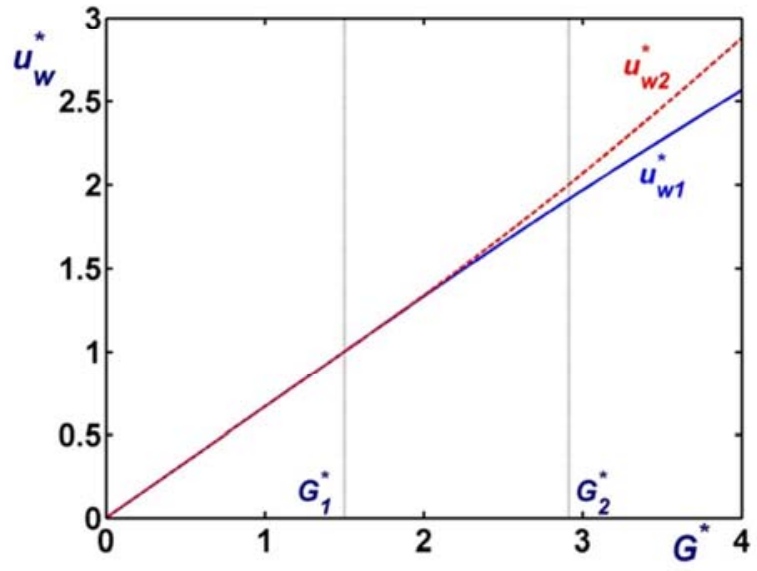

(b)

Figure 5. Wall shear stresses and slip velocities as functions of the pressure gradient when $n=1 / 2$ and $s=1$ : (a) $B_{1}=0.001$ and $B_{2}=0.002$ (weak slip) in which case $G_{1}^{*}=1.5$ and $G_{2}^{*}=2.1752 ;$ (b) $B_{1}=1$ and $B_{2}=2$ (strong slip) in which case $G_{1}^{*}=1.5$ and $G_{2}^{*}=2.9129$. The vertical lines indicate the two critical values of the pressure gradient. 


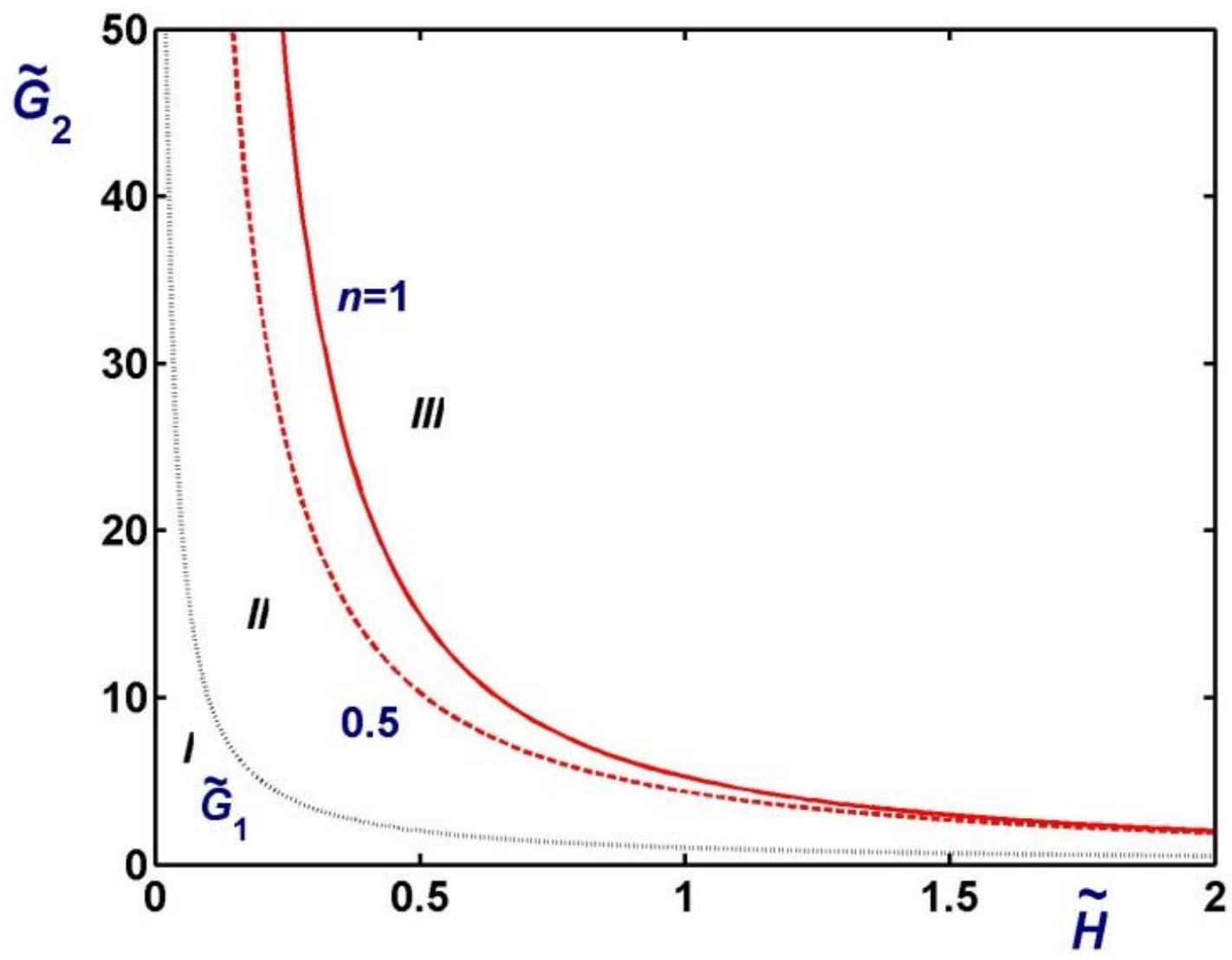

Figure 6. Effect of the power-law exponent $n$ on the second critical pressure gradient $\tilde{G}_{2}$ for $\kappa=0$ (no-slip at the lower wall) and $s=1$ (Navier-slip at the upper wall). The solid curve has been obtained with $n=1$ (Bingham fluid) and the dashed one with $n=1 / 2$ (Herschel-Bulkley). The lower dotted line is the plot of $\tilde{G}_{1}$, which is independent of the value of exponent $n$. The region between the curves of $\tilde{G}_{1}$ and $\tilde{G}_{2}$ corresponds to Regime II, while the area below the curve of $\tilde{G}_{1}$ corresponds to Regime I (full-slip flow). 


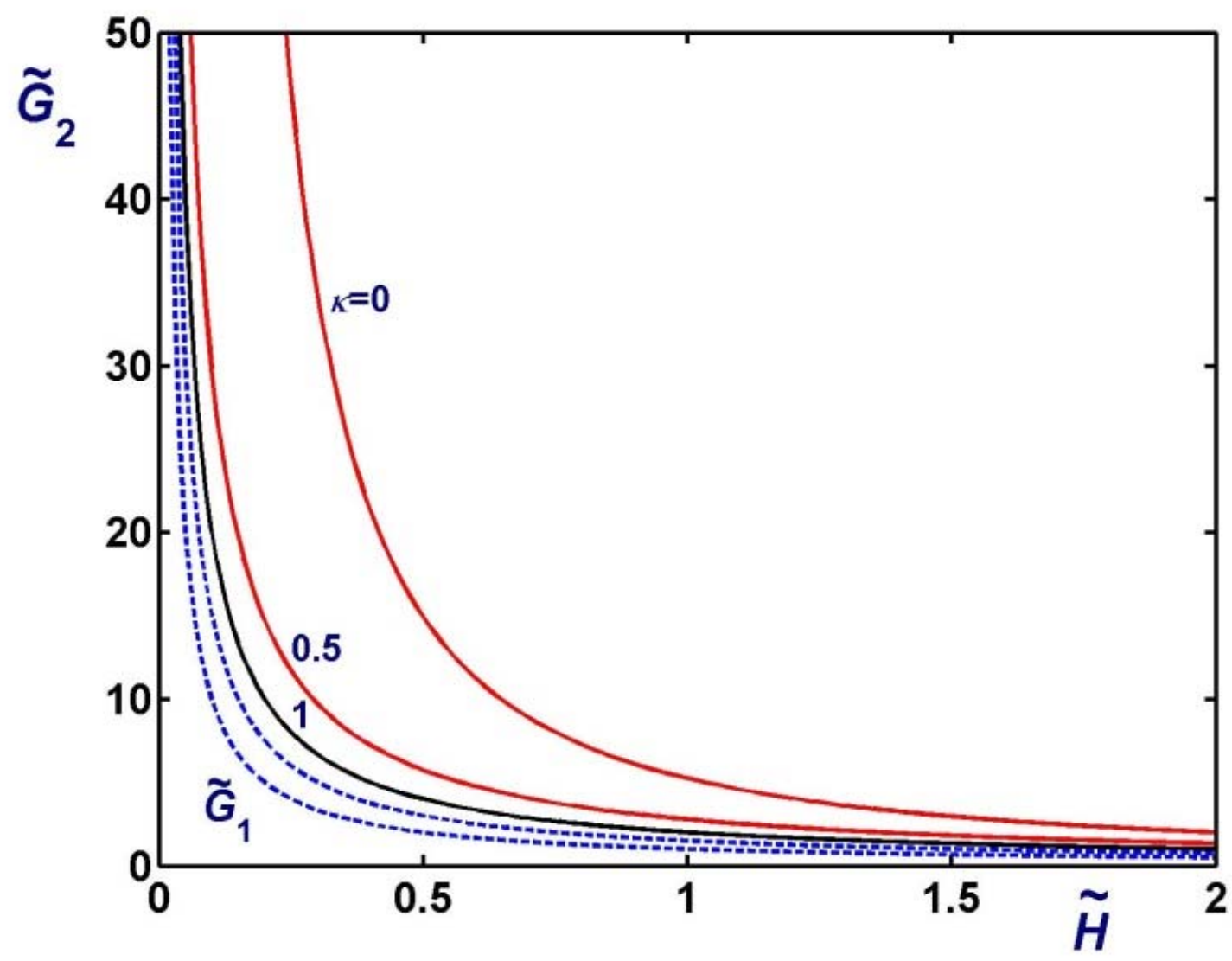

Figure 7. Effect of the slip number ratio $\kappa \equiv B_{1} / B_{2}$ on the critical pressure gradients $\tilde{G}_{1}$ (dashed) and $\tilde{G}_{2}$ (solid) and the different flow regimes for $n=1 / 2$ (Herschel-Bulkley flow). The area between $\tilde{G}_{1}$ and $\tilde{G}_{2}$ corresponds to Regime II, while the area below the curve of $\tilde{G}_{1}$ corresponds to Regime I (full-slip flow). When $\kappa=1$ slip is symmetric and thus $\tilde{G}_{1}$ and $\tilde{G}_{2}$ coincide. 



Figure 8. (a) Effect of the slip parameters on the variation of the asymmetry parameter $S$ with $\bar{U}_{s}$ when $n=1 / 2 ; B_{1}=1$ is kept constant and the ratio $B_{1} / B_{2}$ is varied by varying $B_{2}$. All curves approach asymptotically the dashed line $S=4 \bar{U}_{s} / 3$ as the pressure gradient is increased. (b) Effect of the power-law exponent for $s=1$ (Navier slip) with $B_{1}=1$ and $B_{2}=2$. As the pressure gradient is increased, the results for $n<S$ (i.e. for $n=1 / 2$ and 1/4) approach asymptotically the corresponding dashed lines $S=2^{1 / n} \bar{U}_{s} /(1+1 / n)$, while the results for $n=s=1$ converge to the point $\left(\bar{U}_{s \infty}, S_{s \infty}\right)=(1 / 8,1 / 8)$. 




(a)

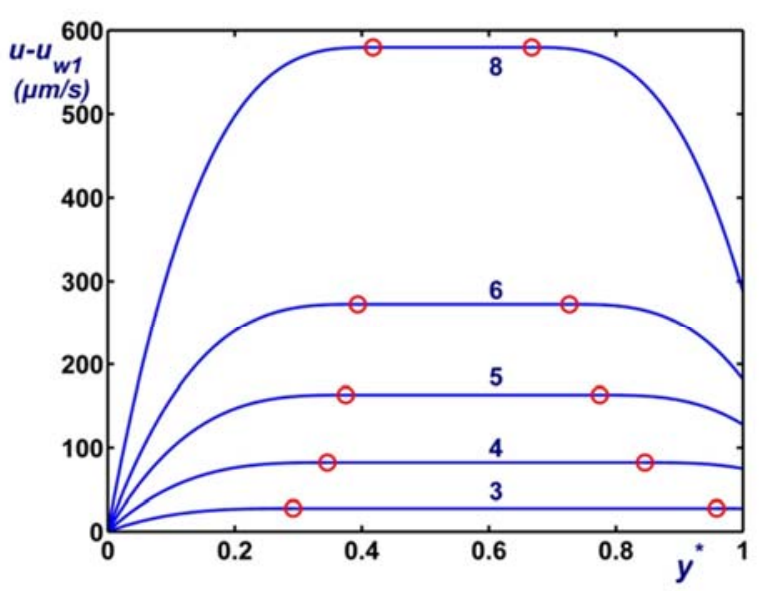

(c)

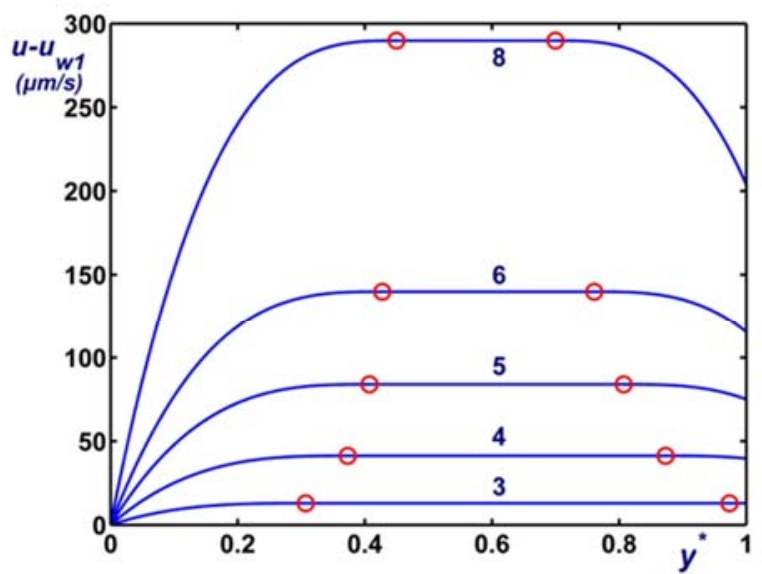

(b)



(d)

Figure 9. Velocity profiles for different channel gaps and various dimensionless pressure for $n=1 / 2$, $k=4.1 \mathrm{~Pa} \mathrm{~s}^{1 / 2}, \tau_{0}=11.2 \mathrm{~Pa}, \beta_{1}=10^{5} \mathrm{~Pa} \mathrm{~m}^{-1} \mathrm{~s}, \beta_{2}=0.5310^{5} \mathrm{~Pa} \mathrm{~m}^{-1} \mathrm{~s}$, and $s=1$ (taken from the data of Vayssade et al [27]): (a) $h=7 \mu \mathrm{m}$ with $B_{1}=2.1441, B_{2}=4.0455$, and $G_{2}^{*}=2.8530$; (b) $h=20 \mu \mathrm{m}$ with $B_{1}=0.7504, B_{2}=1.4159$, and $G_{2}^{*}=2.8044$; (c) $h=50 \mu \mathrm{m}$ with $B_{1}=0.3002, B_{2}=0.5664$, and $G_{2}^{*}=2.7291 ;$ (d) $h=100 \mu \mathrm{m}$ with $B_{1}=0.1501, B_{2}=0.2832$, and $G_{2}^{*}=2.6534 . G_{1}^{*}=1.53$ in all cases. 


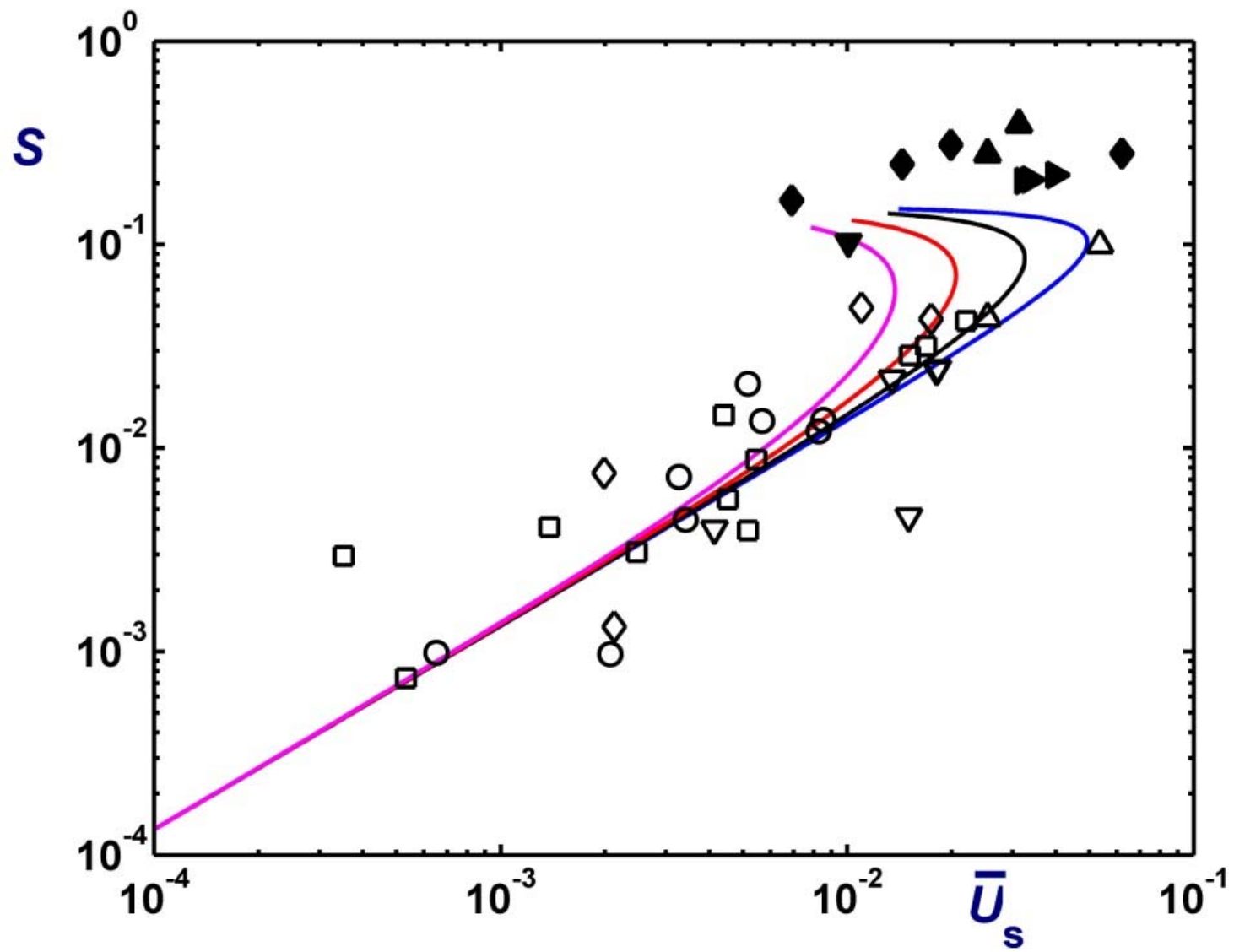

Figure 10. Asymmetry parameter $S$ versus slip parameter $\bar{U}_{s}$ for $n=1 / 2, s=1$ and $h=7 \mu \mathrm{m}$ (rightmost curve), $20 \mu \mathrm{m}, 50 \mu \mathrm{m}$, and $100 \mu \mathrm{m}$ (leftmost curve) compared with experimental data for $h=7 \mu \mathrm{m}$ $(\boldsymbol{\Delta}, \Delta), 20 \mu \mathrm{m}(\diamond, \diamond), 50 \mu \mathrm{m}(\square), 75 \mu \mathrm{m}(\boldsymbol{\nabla}, \nabla), 80 \mu \mathrm{m}(\bullet)$ and $100 \mu \mathrm{m}(\mathrm{o})$. Solid symbols correspond to Regime II (semi-plateau velocity profiles) and open symbols to Regime III (asymmetric velocity profiles). 